\title{
Search for polyoma-, herpes-, and bornaviruses in squirrels of the family Sciuridae
}

Vanessa Schulze ${ }^{1}$, Peter W. W. Lurz ${ }^{2}$, Nicola Ferrari ${ }^{3}$, Claudia Romeo ${ }^{3}$, Michael A. Steele ${ }^{4}$, Shealyn Marino ${ }^{4}$, Maria Vittoria Mazzamuto ${ }^{5}$, Sébastien Calvignac-Spencer ${ }^{6}$, Kore Schlottau ${ }^{7}$, Martin Beer ${ }^{7}$, Rainer G. Ulrich ${ }^{1,8^{*}}$ and Bernhard Ehlers ${ }^{9 *}$

\begin{abstract}
Background: Squirrels (family Sciuridae) are globally distributed members of the order Rodentia with wildlife occurrence in indigenous and non-indigenous regions (as invasive species) and frequent presence in zoological gardens and other holdings. Multiple species introductions, strong inter-species competition as well as the recent discovery of a novel zoonotic bornavirus resulted in increased research interest on squirrel pathogens. Therefore we aimed to test a variety of squirrel species for representatives of three virus families.

Methods: Several species of the squirrel subfamilies Sciurinae, Callosciurinae and Xerinae were tested for the presence of polyomaviruses (PyVs; family Polyomaviridae) and herpesviruses (HVs; family Herpesviridae), using generic nested polymerase chain reaction (PCR) with specificity for the PyV VP1 gene and the HV DNA polymerase (DPOL) gene, respectively. Selected animals were tested for the presence of bornaviruses (family Bornaviridae), using both a broad-range orthobornavirus- and a variegated squirrel bornavirus 1 (VSBV-1)-specific reverse transcriptionquantitative PCR (RT-qPCR).

Results: In addition to previously detected bornavirus RNA-positive squirrels no more animals tested positive in this study, but four novel PyVs, four novel betaherpesviruses (BHVs) and six novel gammaherpesviruses (GHVs) were identified. For three PyVs, complete genomes could be amplified with long-distance PCR (LD-PCR). Splice sites of the PyV genomes were predicted in silico for large T antigen, small T antigen, and VP2 coding sequences, and experimentally confirmed in Vero and $\mathrm{NIH} / 3 T 3$ cells. Attempts to extend the HV DPOL sequences in upstream direction resulted in contiguous sequences of around 3.3 kilobase pairs for one BHV and two GHVs. Phylogenetic analysis allocated the novel squirrel PyVs to the genera Alpha- and Betapolyomavirus, the BHVs to the genus Muromegalovirus, and the GHVs to the genera Rhadinovirus and Macavirus.

(Continued on next page)
\end{abstract}

\footnotetext{
* Correspondence: rainer.ulrich@fli.de; ehlersb@rki.de

${ }^{1}$ Institute of Novel and Emerging Infectious Diseases,

Friedrich-Loeffler-Institut, Greifswald - Insel Riems, Germany

${ }^{9}$ Division 12 'Measles, Mumps, Rubella and Viruses Affecting Immunocompromised Patients', Robert Koch-Institute, Berlin, Germany

Full list of author information is available at the end of the article
}

(C) The Author(s). 2020 Open Access This article is licensed under a Creative Commons Attribution 4.0 International License, which permits use, sharing, adaptation, distribution and reproduction in any medium or format, as long as you give appropriate credit to the original author(s) and the source, provide a link to the Creative Commons licence, and indicate if changes were made. The images or other third party material in this article are included in the article's Creative Commons licence, unless indicated otherwise in a credit line to the material. If material is not included in the article's Creative Commons licence and your intended use is not permitted by statutory regulation or exceeds the permitted use, you will need to obtain permission directly from the copyright holder. To view a copy of this licence, visit http://creativecommons.org/licenses/by/4.0/ The Creative Commons Public Domain Dedication waiver (http://creativecommons.org/publicdomain/zero/1.0/) applies to the data made available in this article, unless otherwise stated in a credit line to the data. 
(Continued from previous page)

Conclusions: This is the first report on molecular identification and sequence characterization of PyVs and HVs and the detection of bornavirus coinfections with PyVs or HVs in two squirrel species. Multiple detection of PyVs and $\mathrm{HVs}$ in certain squirrel species exclusively indicate their potential host association to a single squirrel species. The novel PyVs and HVs might serve for a better understanding of virus evolution in invading host species in the future.

Keywords: Bornavirus, Polyomavirus, Betaherpesvirus, Gammaherpesvirus, Large T, Small T, VP2, Splicing, Squirrel

\section{Background}

Squirrels (family Sciuridae) are members of the order Rodentia, and with the exception of Antarctica, distributed globally on all continents. They occur both in the Old and in the New world, and squirrel species diversity is particularly high in Africa and Asia [1]. Squirrels are generally distinguished as ground, flying (gliding) or tree squirrels. With respect to the latter, the Eurasian red squirrel (Sciurus vulgaris) is the dominant squirrel species across the Palaearctic $[2,3]$. In contrast to the North American red or pine squirrel (Tamiasciurus hudsonicus) which is a boreal coniferous species [1], the Eurasian red squirrel originally occupied all available forest habitats in the absence of other tree squirrels across most of its range.

The Eastern grey squirrel (Sciurus carolinensis) is a broadleaf specialist and was originally distributed in the eastern deciduous forests of Northern America [4]. However, the species has been translocated repeatedly within North America and globally to Europe, South Africa and Australia (e.g. see $[4,5])$. The multiple introductions and subsequent translocations had devastating consequences for local Eurasian red squirrel populations in deciduous and mixed forest landscapes in Great Britain, Ireland and Italy leading to large-scale declines as a result of competition for resources and introduced pathogens [6-8]. With regard to pathogens, the replacement of native Eurasian red squirrels by the Eastern grey squirrels in the British Isles is highly accelerated by a squirrelpox virus (e.g. [9]). However, the resulting research interest on pathogens also resulted in the identification of other potential disease threats to red squirrels such as leprosy bacilli [10] and adenovirus [11-13]. Although squirrel adenovirus has been known for many years, it was only recently that the complete genome of squirrel adenovirus 1 (SqAdV-1) was determined and found to indicate a close relationship between British and Continental European red squirrel populations [11, 13].

Prevost's squirrels (Callosciurus prevostii) and Pallas's squirrels (Callosciurus erythraeus) belong to the subfamily Callosciurinae. Both species are native to South-East Asia and Pallas's squirrels were introduced to Italy, France, Belgium and the Netherlands. They typically represent non-native, invasive tree squirrel species that arrived in Europe via pet trade [14-16]. The variegated squirrel (Sciurus variegatoides), a species of dry tropical forests in Central America, is another tree squirrel that appears popular among squirrel breeders [17], perhaps due to its great variability in coat colour [18]. Whilst there has been some progress in recognition of potential threats to wildlife and people from introduced species (e.g. [19, 20]), little information is available about viral and bacterial pathogens and parasites in squirrels and the role of squirrels in population dynamics, competitive interactions or as reservoirs for zoonotic diseases.

Recently a novel zoonotic bornavirus, variegated squirrel bornavirus 1 (VSBV-1), was detected in five different squirrel species from private holdings and zoos, including Sciurus variegatoides and Callosciurus prevostii and is associated with cases of fatal encephalitis of their breeders and care takers [17, 21-23]. Bornaviruses (family Bornaviridae) are enveloped spherically structured viruses with a single-stranded RNA genome of negative polarity (size around 8.9 kilobases (kb)). Bornaviruses have been identified in a wide range of hosts, e.g. mammals, birds and reptiles [24]. Members of the genus Orthobornavirus infecting mammals are assigned to two species: Mammalian 1 orthobornavirus (Borna Disease Virus 1 and 2; BoDV-1 and BoDV-2) and Mammalian 2 orthobornavirus (VSBV-1). These viruses have pathogenic potential for humans and other mammals. BoDV1 is the causative agent of Borna disease, an often fatal neurologic condition of horses, sheep and other domestic mammals. Recently, its zoonotic potential has been demonstrated by molecular and immunohistochemical detection of several BoDV-1-induced fatal encephalitis cases in humans [25-28].

Polyomaviruses (PyVs; family Polyomaviridae) and herpesviruses (HVs; family Herpesviridae) both comprise a plethora of viruses many of which are pathogenic for humans and animals. In members of some mammalian orders (e.g. primates, artiodactyls or rodents) many PyVs and HVs have been identified, some of which are well studied [29-33]. Despite this knowledge, information on PyVs and HVs is still scarce for many mammalian families. This holds particularly true for the family Sciuridae. To our knowledge, there is no report describing the occurrence of PyVs in members of the Sciuridae. From the 1980s there exist descriptions of HVs in a thirteenlined ground squirrel (Ictidomys tridecemlineatus) [34], 
and Citellus spp. (now Spermophilus) [35, 36] but these reports are based only on electron microscopy and cytopathogenic effects in infected cell cultures, which were assumed to be typical for HVs, despite the lack of sequence data.

PyVs are small non-enveloped viruses with an icosahedral capsid and a circular double-stranded DNA genome that consists of approximately 5 kilobase pairs (kbp). The family Polyomaviridae comprises around 100 members, including 14 that infect humans. PyVs have been found in many hosts, including humans, nonhuman primates, rodents, cattle, bats, birds, and fish [31]. PyVs cause subclinical infections, as well as acute systemic diseases, the latter mainly in immune-compromised individuals. Some PyVs have transforming activity in vitro and reveal tumorigenic capacity in laboratory animals. Merkel cell polyomavirus (MCPyV) is the first human PyV that is associated with a tumor in humans [37, 38].

The genomic organization of mammalian PyVs comprises three regions: The early transcriptional region, the late transcriptional region and the non-coding control region (NCCR). The early region encodes regulatory proteins, including large T-antigen (LTAg) and small Tantigen (STAg). The counter clock-wise oriented late region encodes the structural proteins VP1, VP2, and VP3. Early and late regions are separated by the NCCR, which controls DNA replication and transcription from the early and late promoters [39]. LTAg and STAg are involved in viral transcription and replication. LTAg induces the synthesis phase of cells and can cause an abnormal stimulation of the cell cycle and tumor formation $[31,39,40]$. Mammalian PyVs are assigned to three distinct genera within the family Polyomaviridae: Alpha-, Beta-, and Deltapolyomavirus [41].

HVs are a family of large, enveloped viruses with a double-stranded DNA genome (length: 110-295 kbp) that infect many vertebrates, including humans and nonhuman primates [29]. Mammalian HVs are divided into three distinct subfamilies within family Herpesviridae: Alphaherpesvirinae, Betaherpesvirinae (BHVs) and Gammaherpesvirinae (GHVs). All HVs share the capacity to establish a state of latency resulting in lifelong association with the infected host. After reactivation, an infectious virus is produced and spreads to other susceptible individuals $[29,42]$. Herpesviruses cause a variety of diseases in humans and animals, including some cancers.

As bornaviruses have so far only been identified in five of the $>280$ sciurid species, and sciurid PyVs and HVs are not yet known, we sought to improve our knowledge on such viruses in the Sciuridae family and performed a molecular survey in squirrels of different species and subfamilies. Spleen and lung samples of 238 animals from five countries (Canada, USA, Italy, UK and Germany) were tested with generic nested PCRs [43-49] for the identification of PyVs and HVs. Brain samples from 126 of these animals originating from four countries (Canada, USA, Italy and UK) were analyzed with broad-range orthobornavirus RT-qPCR for the generic detection of orthobornaviruses and a VSBV-1-specific reverse transcription-quantitative polymerase chain reaction (RT-qPCR). In case of successful PyV sequence detection, we aimed at generating complete genome sequences, and in case of HV sequence detection, we wanted to amplify and sequence a genome segment, which comprises around $3.4 \mathrm{kbp}$ and extends from the glycoprotein $\mathrm{B}(\mathrm{gB})$ gene to the DNA polymerase (DPOL) gene. This approach led to the discovery of four PyVs and 10 HVs (four BHVs and six GHVs).

\section{Methods}

\section{Sample collection and nucleic acid preparation}

Squirrels $(n=126)$ of five species belonging to the family Sciuridae were collected in four different countries (Canada, USA, Italy and UK), dissected according to standard protocols and brain, lung and spleen samples of these animals were available for screening for all three viruses. In addition, lung and spleen samples from another 112 squirrels belonging to another three species were available from previous bornavirus studies $[17,21,22]$. Thus, a total of 361 organ samples (243 spleen samples and 118 lung samples) were available for PyV and HV analyses (Tables 1, 2 and 3).

RNA extraction was performed using the KingFisher $^{\mathrm{rm}}$ Flex Purification System (Thermo Fisher) in combination with the Nucleo Mag Vet Kit (Macherey Nagel) following the instructions of the manufacturer. DNA extraction was performed using EURx GeneMatrix Tissue DNA Purification Kit (Roboklon), Qiagen DNeasy Blood \& Tissue-Kit (Qiagen) and the KingFisher $^{\mathrm{Tm}}$ Flex Purification System (Thermo Fisher) in combination with the Nucleo Mag Vet Kit (Macherey Nagel). DNA preparations were stored at $-20^{\circ} \mathrm{C}$ and RNA preparations at $-80^{\circ} \mathrm{C}$. During the nucleic extraction processes negative controls were carried along to monitor for potential contaminations. Morphological species identification was confirmed for all samples by cytochrome $b$ PCR and sequencing according to a previously described protocol ( [50], data not shown). Prior to nucleic acid extraction no attempts concerning virus isolation in cell culture were done.

\section{Bornavirus screening of squirrel brain samples}

Brain samples of the newly dissected 126 squirrels were screened with broad-range and VSBV-1 specific RT-qPCRs (Additional file 1) as described previously $[17,28]$. The other animals $(n=112)$ have already been investigated before [17, 21, 22]. 
Table 1 Bornavirus reverse transcription - quantitative polymerase chain reaction (RT-qPCR) analysis of squirrels from wildlife and holdings

\begin{tabular}{|c|c|c|c|}
\hline $\begin{array}{l}\text { Host taxonomic name } \\
\text { (subfamily, species) }\end{array}$ & Host common name & $\begin{array}{l}\text { n positive/ total } n \text { tested } \\
\text { in this study }\end{array}$ & $\begin{array}{l}\text { RT-qPCR positive/ total } n \\
\text { tested in previous studies } \\
{[17,21,22]}\end{array}$ \\
\hline \multicolumn{4}{|l|}{ Sciurinae } \\
\hline Sciurus carolinensis & Eastern grey squirrel & $0 / 77^{\mathrm{a}}$ & $0 / 11^{a}$ \\
\hline Sciurus variegatoides & Variegated squirrel & & $7 / 7^{b}$ \\
\hline Sciurus vulgaris & Eurasian red squirrel & & $0 / 77^{a}$ \\
\hline Tamiasciurus hudsonicus & American red squirrel & $0 / 1^{a}$ & \\
\hline \multicolumn{4}{|l|}{ Xerinae } \\
\hline Urocitellus richardsonii & Richardson's ground squirrel & $0 / 11^{a}$ & \\
\hline Tamias striatus & Eastern chipmunk & $0 / 2^{a}$ & \\
\hline \multicolumn{4}{|l|}{ Callosciurinae } \\
\hline Callosciurus erythraeus & Pallas's squirrel & $0 / 35^{a}$ & \\
\hline Callosciurus prevostii & Prevost's squirrel & & $10 / 17^{b}$ \\
\hline total & & $0 / 126$ & 17/112 \\
\hline
\end{tabular}

all individuals originated from wildlife

ball individuals originated from holdings

Identification of polyomaviruses and herpesviruses with generic PCR assays

For identification of PyVs and HVs generic nested PCRs were performed, that broadly detect a partial VP1 coding sequence (CDS) of PyVs (Additional files 1 and 2) or a fragment of the DPOL gene of $\mathrm{HVs}$ (Open reading frame 09 (ORF09) of GHV; ORF UL54 of BHV) (Additional file 1 and Additional file 3 ) in the second PCR round. Both nested PCRs were performed as carried out previously $[46,48]$.

\section{Amplification of $\mathrm{gB}$ sequences of betaherpesviruses and gammaherpesviruses with generic PCR}

For amplification of gB sequences of BHVs (ORF UL55) and GHVs (ORF08), we used subfamily-specific nested primer sets (Additional file 1) essentially as described previously $[51,52]$. The scheme of multi-level PCR analysis used for squirrel BHVs and GHVs is shown in Additional file 3.

\section{Nested long-distance PCR with virus-specific primers}

For all PyVs, specific nested primers (Additional file 1) were selected tail-to-tail from the sequences amplified with generic PCR. They were used for the amplification and sequencing of the remaining parts of the circular genomes (approximately $5 \mathrm{kbp}$ ). For all $\mathrm{HVs}$, for which both $\mathrm{gB}$ and DPOL sequences could be amplified, nested primers (Additional file 1) were selected that were specific for each virus and used in long-distance PCR (LD-PCR) for the amplification and sequencing of the sequence that spans the gap between the partial $\mathrm{gB}$ and DPOL sequences. LD-PCR was performed with the TaKaRa-Ex PCR system (Takara Bio Inc.), according to the manufacturer's instructions. After the first PCR round, a $2 \mu \mathrm{l}$ aliquot of the reaction mix was used as template in the second-round reaction.

\section{Hemi-nested herpesvirus DPOL PCR}

In cases where only the generic HV PCR was successful (and not gB PCR and/or LD-PCR), the short DPOL sequence was extended in upstream direction with heminested PCR, using the outer sense primer (285 DFA) of the generic HV PCR and two virus-specific antisense primers for amplification of approximately 480 base pairs (bp) (Additional file 1). The hemi-nested DPOL PCR was carried out as described above for generic HV PCR.

Based on these extended sequences we were able to design again virus-specific primers that were used for rescreening of all samples of the respective species.

\section{Nested PCR with polyomavirus-specific primers}

For all novel PyVs, for which full genomes could be assembled, nested primer sets (Additional file 1) were selected. They were used for amplification of sequences of approximately $800 \mathrm{bp}$ that encompass the short overlap between the sequences generated from the generic PCR fragments and the LD-PCR fragments of the respective PyV genome. PCR was performed in a total volume of $25 \mu \mathrm{l}$ with $0.4 \mu \mathrm{l}$ ( 2 units) Applied Biosystems AmpliTaq Gold DNA Polymerase (Thermo Fisher), 25 pmol of each primer, $200 \mu \mathrm{M}$ dideoxynucleoside triphosphates (dNTPs), $2 \mathrm{mM} \mathrm{MgCl}_{2}, 5 \%$ dimethyl sulfoxide (DMSO) and $250 \mathrm{ng}$ of sample DNA as template. A thermocycler from Biometra was used under the following cycling conditions: activation of the polymerase at $95^{\circ} \mathrm{C}$ for $12 \mathrm{~min}$ and 45 cycles of denaturation at $95^{\circ} \mathrm{C}$ for 30 $\mathrm{s}$, annealing at $61^{\circ} \mathrm{C}$ for $30 \mathrm{~s}$, and elongation at $72^{\circ} \mathrm{C}$ for $5 \mathrm{~min}$, followed by a final extension step at $72^{\circ} \mathrm{C}$ for 30 


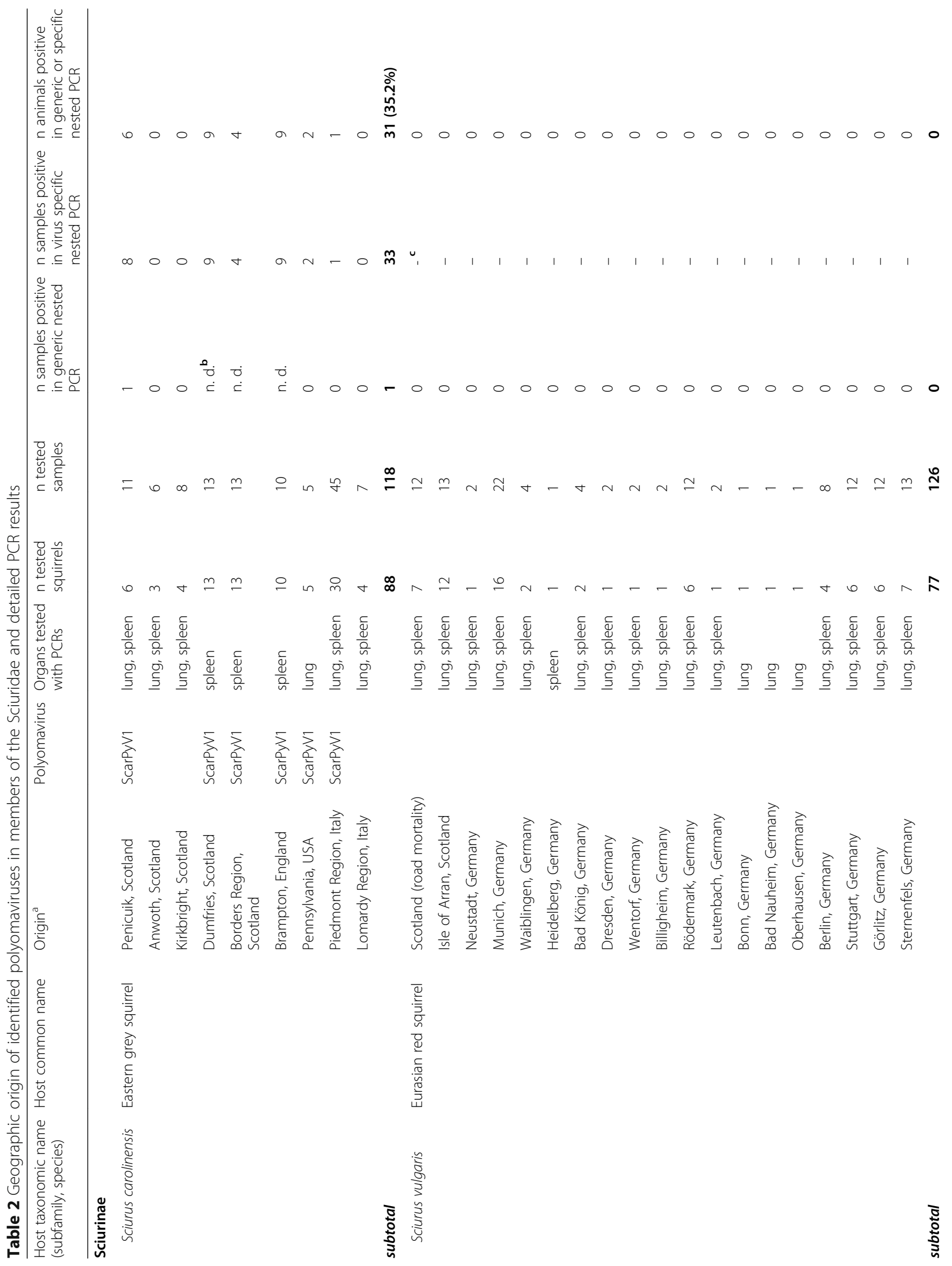




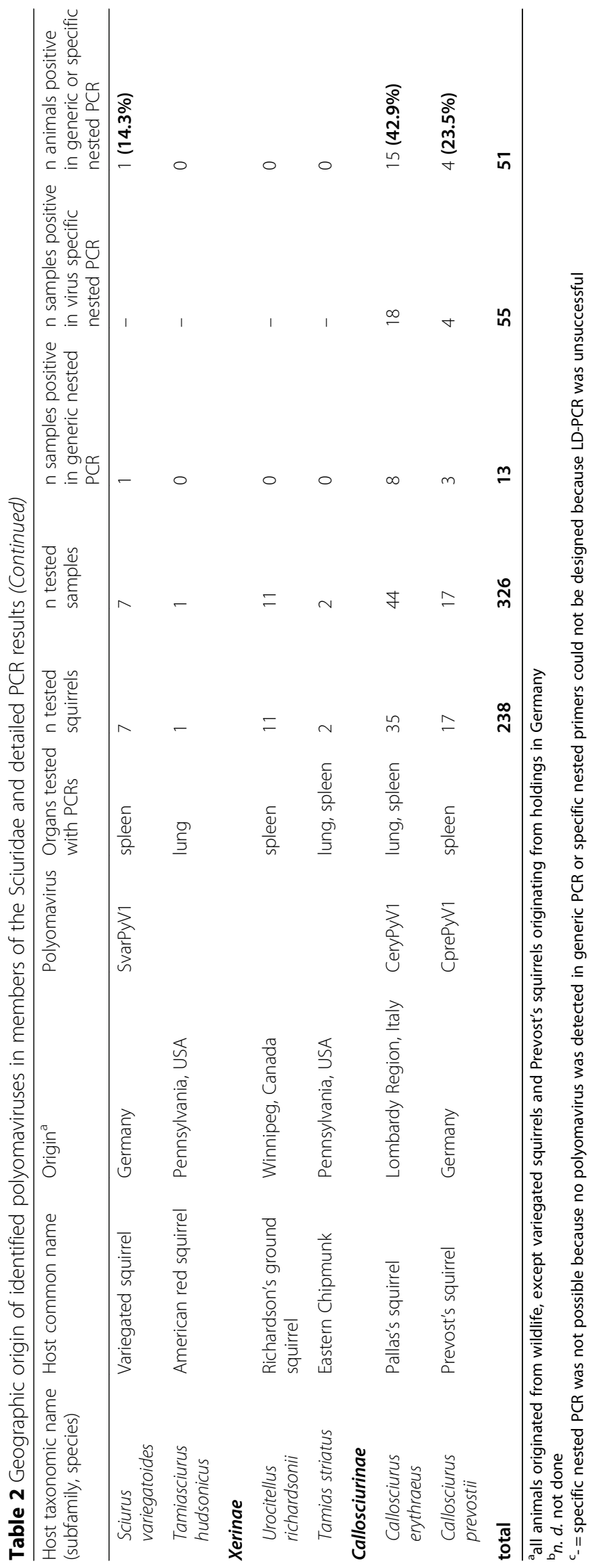




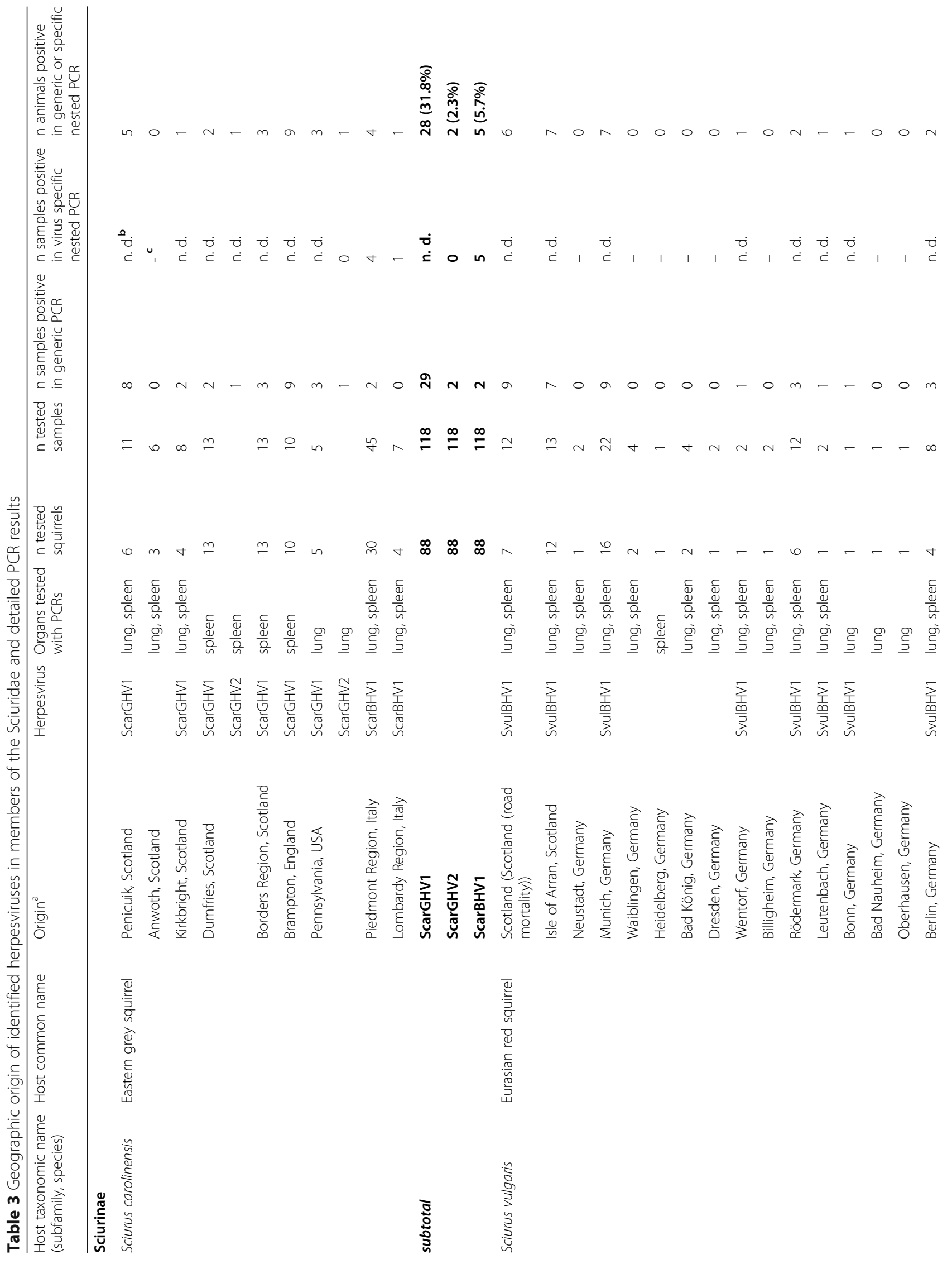


Schulze et al. Virology Journal

(2020) 17:42

Page 8 of 21

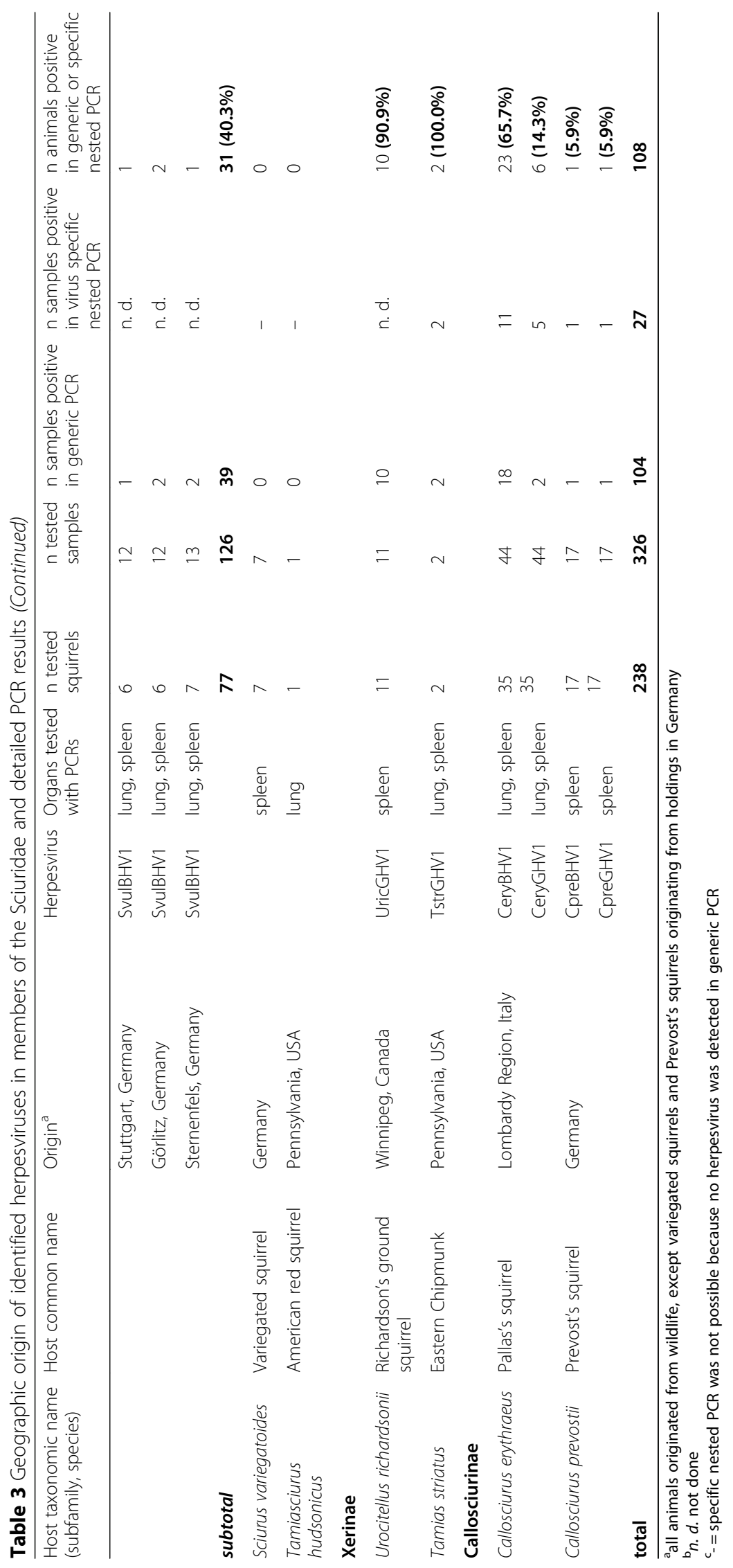


min. For the second PCR round, a $1 \mu$ l aliquot of the first-round reaction mix was used as a template. The primers were also used for more sensitive re-screening all samples of the respective host species.

\section{Nested PCR with herpesvirus-specific primers}

For all novel HVs, for which extended sequences could be determined, nested primer sets were selected for rescreening all samples of the respective host species (Additional file 1). They were used as described above for specific PyV amplification (annealing temperatures listed in Additional file 1).

\section{RT-PCR and PCR controls}

For each round of all nested PCRs and the RT-qPCR of our screening, no-template controls (PCR-grade $\mathrm{H}_{2} \mathrm{O}$ ) and extraction controls were carried along to detect any possible contaminations. These analyses were negative for all PCRs and RT-qPCRs. As positive PCR controls, DNA extracts of samples were used that tested positive for BHVs, GHVs or PyVs in previous studies.

\section{PCR product purification and sequencing}

All PCR products were purified with $\mathrm{MSB}^{\star}$ Spin PCRapace (Stratec), according to the manufacturer's instruction and directly sequenced using the BigDye Terminator v3.1 system (Life Technologies) on an Applied Biosystems 3500xL DX Genetic Analyzer (Thermo Fisher). The LD-PCR products were sequenced by a classical primer walking strategy (primers not listed).

\section{Synthesis of polyomavirus early and late region}

Early or late region plus flanking sequences (approximately $3.1 \mathrm{kbp}$ ) of Sciurus carolinensis polyomavirus 1 (ScarPyV1; GenBank accession number MK671101) were commercially synthesized and delivered as recombinant plasmids (Biomatik). They were named pScarPyV1early and pScarPyV1late and transformed into competent Escherichia coli DH5 alpha cells (Thermo Fisher). Plasmid DNA was extracted with Invisorb ${ }^{\oplus}$ Spin Plasmid Mini Two (Stratec) according to the manufacturer's instruction.

\section{Cell lines}

Vero cells C1008 (monkey kidney cells; European Collection of Authenticated Cell Cultures (ECACC) \# 85020206) were cultured in standard high-glucose Dulbecco's minimal Eagle medium (DMEM, Thermo Fisher) containing $10 \%$ fetal calf serum (FCS) (PAN Biotech) and 1\% penicillin/streptomycin (Thermo Fisher). For NIH/3T3 cells (mouse embryo fibroblast cells; American Tissue Culture Collection, ATCC ${ }^{\oplus}$, CRL-1658 $8^{\text {тM }}$ ) the same medium was used, except that 5\% FCS (PAN Biotech) was added. Both cell lines were cultivated at $37^{\circ} \mathrm{C}$ and $5 \% \mathrm{CO}_{2}$. DNA extracts of cell aliquots were tested with PCR for absence of mycoplasma contamination [53]. Primers are listed in Additional file 1.

\section{Transfection of cells}

As described previously [54], cells were transfected with $1 \mu \mathrm{g}$ DNA of plasmid pScarPyV1early or plasmid pScarPyV1late, using X-tremeGENE HP DNA Transfection Reagent (Roche Applied Biosciences). Before transfection, Vero cells were seeded in a volume of $500 \mu \mathrm{l}$ cell culture medium in tissue culture plates with 24 wells (Sarstedt). For NIH/3T3 cells, Cell+ plates with 24 wells and a special Cell+ growth surface for sensitive adherent cells (Sarstedt) were used. Transfection procedures were performed $24 \mathrm{~h}$ after seeding according to the manufacturer's instructions.

\section{RNA extraction and cDNA synthesis}

Total RNA was isolated on $0,1,2.5$, and 6 days post transfection of recombinant plasmid DNA using the NucleoSpin ${ }^{\oplus}$ RNA-Kit (Macherey Nagel) according to the manufacturer's instructions. DNA was removed by an additional Turbo DNA-free DNase treatment (Thermo Fisher). RNA concentrations were determined with the NanoDrop 8000 device (Thermo Fisher) at $260 \mathrm{~nm}$. Synthesis of cDNA was carried out with 500 ng RNA using SuperScript II Reverse Transcriptase (Thermo Fisher) and oligo(dT)16 primers (Roche Applied Bioscience).

\section{PCR for identification of introns}

To identify introns in the early and late region of ScarPyV1, PCR was performed with cDNA as a template, using primers specific for ScarPyV1 that bind in flanking regions of predicted introns (Additional file 1). PCR was performed with $2.5 \mu \mathrm{l} \mathrm{cDNA}$ in a total volume of $25 \mu \mathrm{l}$ with $0.2 \mu \mathrm{l}$ (1 unit) Applied Biosystems AmpliTaq Gold DNA Polymerase (Thermo Fisher), $1 \mu \mathrm{M}$ of each primer, $200 \mu \mathrm{M}$ dNTP PCR Mix (Metabion), $2 \mathrm{mM} \mathrm{MgCl}_{2}$ and 5\% DMSO (Merck). All PCR products were purified with $\mathrm{MSB}^{\circ}$ Spin PCRapace (Stratec), according to the manufacturer's instructions. Sequencing reactions were performed as described above.

\section{Bioinformatics and phylogenetic analysis}

For the datasets we selected reference viral genomes representing all currently recognized species in the family/subfamily considered as well as additional viruses whose genomes represented distinct viral lineages discussed in the literature but still not integrated into the official taxonomy (sensu International Committee on Taxonomy of Viruses (ICTV)). For PyVs, this represented 109 viruses; for BHVs, 21 viruses; and for GHVs, 39 viruses. We extracted the LTAg and VP1 (PyV) or DPOL and gB (BHV and GHV) coding sequences from these genomes as well as from the novel viruses we 
identified in squirrels using Geneious v11.1.5 [55]. For each coding sequence, sequences were translated into amino acid sequences and aligned using Muscle [56] as implemented in Seaview v4 [57]. Conserved amino acid blocks were then selected using Gblocks as implemented in Seaview, using options for a less stringent selection: allow smaller final blocks, allow gap positions within the final blocks and allow less strict flanking positions [58]. The final amino acid sequence alignments comprised 260 (VP1), 517 (LTAg), 628 (DPOL BHV), 298 (gB BHV), 622 (DPOL GHV) and 282 (gB GHV) positions, respectively.

For phylogenetic analyses of all datasets we first ran Maximum-Likelihood (ML) analyses using PhyML v3 with smart model selection (PhyML-SMS) using the Bayesian information criterion and a tree search using subtree pruning and regrafting [57, 59,60]. Branch robustness was estimated using Shimodaira-Hasegawa-like approximate likelihood ratio tests (SH-like aLRT) [61]. The PyV and GHV ML trees were rooted with TempEst v1.5 by minimizing the variance of root-to-tip distances [62]; the BHV ML trees were rooted using roseolovirus outgroups. We then ran Bayesian Markov chain Monte Carlo (BMCMC) runs using BEAST v1.10.4 [63]. For each alignment, we used the amino acid substitution model identified by PhyML-SMS, an uncorrelated relaxed clock (lognormal) model and a speciation model (birth-death) as a tree prior. The output of multiple BMCMC runs was examined for convergence and appropriate sampling of the posterior using Tracer v1.7.1 [64], before being merged using LogCombiner v1.10.4 (distributed with BEAST). The maximum clade credibility (MCC) tree was identified from the posterior set of trees (PST) and annotated with TreeAnnotator v1.10.4 (also distributed with BEAST). Branch robustness was estimated based on their posterior probability in the PST.

\section{Results}

\section{Bornavirus analysis}

In previous studies, brain and oral swab samples of 112 squirrels belonging to various species were investigated with a broad-range orthobornavirus RT-qPCR and VSBV1-specific RT-qPCR. VSBV-1-RNA was detected in brain and/or oral swab samples of 7/7 individuals from holdings of variegated squirrels, in samples of 10/17 Prevost's squirrels and a few other species [17, 21,22], but not in any brain samples of the 77 tested Eurasian red squirrels and in any of the 11 Eastern grey squirrels [17, 21, 22]. Here we investigated a total of 126 brain samples from wildlifederived squirrels from four countries, but did not find any positive specimens (Table 1).

Identification of novel polyomaviruses and herpesviruses To identify PyVs and HVs in squirrels, we analyzed 238 squirrels (spleen, $n=208$; lung, $n=118$ ) of eight different species (Tables 2 and 3). First we screened with generic nested PCR that targets a short fragment of the major capsid VP1 CDS of mammalian PyVs (Additional file 1 and Additional file 2) [43, 47-49] and generic nested PCR with specificity for the highly conserved DPOL gene of mammalian HVs (Additional file 1 and Additional file 3) [44-46]. Products of expected length were sequenced and sequences analyzed using Nucleotide Basic Local Alignment Search Tool (BLASTn). Thirteen samples of twelve animals were positive for yet unknown PyVs (Table 2) and 104 samples of 89 animals were positive for yet unknown HVs (Table 3) as revealed by BLASTn analysis (data not shown). In total, four novel PyVs, and 10 novel HVs (four BHVs and six GHVs) were identified and tentatively named: Sciurus carolinensis polyomavirus 1 (ScarPyV1), Sciurus carolinensis betaherpesvirus 1 (ScarBHV1), Sciurus carolinensis gammaherpesvirus 1 and 2 (ScarGHV1 and ScarGHV2), Sciurus variegatoides polyomavirus 1 (SvarPyV1), Sciurus vulgaris betaherpesvirus 1 (SvulBHV1), Callosciurus erythraeus polyomavirus 1 (CeryPyV1), Callosciurus erythraeus betaherpesvirus 1 (CeryBHV1), Callosciurus erythraeus gammaherpesvirus 1 (CeryGHV1), Callosciurus prevostii polyomavirus 1 (CprePyV1), Callosciurus prevostii betaherpesvirus 1 (CpreBHV1), Callosciurus prevostii gammaherpesvirus 1 (CpreGHV1), Urocitellus richardsonii gammaherpesvirus 1 (UricGHV1) and Tamias striatus gammaherpesvirus 1 (TstrGHV1).

In PyV detection with generic PCR, one of 118 spleen samples and one of 88 lung samples from Eastern grey squirrels (Sciurus carolinensis) were positive for ScarPyV1, 1/7 spleen samples of variegated squirrels (Sciurus variegatoides) was positive for SvarPyV1, 8/44 tested samples (6x spleen and $1 \mathrm{x}$ spleen and lung) from $35 \mathrm{Pal}-$ las's squirrels (Callosciurus erythraeus) originating from Italy were positive for CeryPyV1, and 3/17 spleen samples of Prevost's squirrels (Callosciurus prevostii) were positive for CprePyV1 (Table 2).

In $\mathrm{HV}$ tests of 118 spleen and lung samples of Sciurus carolinensis from the UK, Italy and USA with generic PCR, we identified 20 animals from the UK as ScarGHV1-positive (16x in spleen and $4 \mathrm{x}$ in spleen and lung), three Eastern grey squirrels from the USA as ScarGHV1-positive in lung and two individuals as ScarGHV2-positive, one from the UK in spleen and one from the USA in lung. ScarBHV1 was detected in lung samples of two animals from Italy. Testing 77 Sciurus vulgaris revealed 18 SvulBHV1-positive individuals from Germany ( $1 \mathrm{x}$ in spleen, $12 \mathrm{x}$ in lung and $5 \mathrm{x}$ in spleen and lung) and 13 from the UK (1 $\mathrm{x}$ in spleen, $9 \mathrm{x}$ in lung and $3 \mathrm{x}$ in spleen and lung). We identified 10 out of 11 Richardson's ground squirrels (Urocitellus richardsonii) to be positive for UricGHV-1 in spleen and 2/2 Eastern chipmunks (Tamias striatus) positive for TstrGHV1, one in spleen and one in lung. In wild Callosciurus 
erythraeus from Italy as well as Callosciurus prevostii from a holding in Germany, we discovered both a BHV and a GHV. Upon testing a total of 35 Callosciurus erythraeus, we identified 16 individuals as CeryBHV1positive (12x in spleen, $2 x$ in lung and $2 x$ in spleen and lung) and two animals as CeryGHV1-positive ( $1 \mathrm{x}$ in spleen and $1 \mathrm{x}$ in lung). Within the 17 Prevost's squirrels, one spleen each was detected to be positive for CpreBHV1 or CpreGHV1 (Table 3).

We did not detect any PyVs in 77 Eurasian red squirrels, 11 Richardson's ground squirrels and two Eastern chipmunks and no HVs in seven spleen samples of variegated squirrels from German holdings. In one lung sample of an American red squirrel (Tamiasciurus hudsonicus) from the USA, neither PyVs nor HVs were detected (Tables 2 and 3 ).

Based on extended sequences that we generated for three PyVs and eight HVs (see below) we selected specific nested primers (Additional file 1) for more sensitive screening and re-tested all samples of the respective species. By this approach we observed the following prevalences: ScarPyV1 was detected in 35.2\% (31/88 squirrels, $27 \mathrm{x}$ positive in spleen, $2 \mathrm{x}$ lung and $2 \mathrm{x}$ in spleen and lung) of Sciurus carolinensis, SvarPyV1 in 14.3\% (1/7 spleen samples positive) of Sciurus variegatoides, CeryPyV1 in $42.9 \%$ (15/35 squirrels, $12 x$ positive in spleen and $3 x$ in spleen and lung) of Callosciurus erythraeus and CprePyV in $23.5 \%$ (4/17 squirrels positive in spleen) of Callosciurus prevostii (Table 2). For the herpesviruses, the ScarGHV1 prevalence of the Sciurus carolinensis from the UK was $40.8 \%$ (20/49 squirrels, 16x positive in spleen and $4 \mathrm{x}$ in spleen and lung) and for Sciurus carolinensis from the USA prevalence was $60.0 \%$ ( $3 / 5$ spleen samples positive). The second gammaherpesvirus of Sciurus carolinensis (ScarGHV2) was detected in $2.0 \%$ (1/49 squirrels positive in spleen) and $20.0 \%$ (1/5 lung samples) of the samples from Britain and the USA, respectively. The average prevalence of ScarBHV1 amounts 5.7\% (5/88 squirrels positive in spleen) and was only found in Eastern grey squirrels from Italy. The Sciurus vulgaris from the UK revealed a SvulBHV1-prevalence of $68.4 \%$ (13/19 squirrels, $1 \mathrm{x}$ positive in spleen, $9 \mathrm{x}$ in lung and $3 \mathrm{x}$ in spleen and lung) and those from Germany, 31.0\% (18/58 squirrels, $1 \mathrm{x}$ positive in spleen, $12 \mathrm{x}$ in lung and $5 \mathrm{x}$ in spleen and lung). In Callosciurus erythraeus from Italy, the prevalence of CeryBHV1 was $65.7 \%$ (23/35 squirrels, $19 x$ positive in spleen, $1 \mathrm{x}$ in lung and $3 \mathrm{x}$ in spleen and lung) and that of CeryGHV1 was $14.3 \%$ (6/35 squirrels, $5 x$ positive in spleen and $1 \mathrm{x}$ in spleen and lung). Richardson's ground squirrels from Canada showed a very high prevalence $(90.9 \%, 10 / 11$ spleen samples positive) of UricGHV1. The herpesvirus prevalence in Prevost's squirrels was 5.9\%: Two of seventeen samples were positive, one for CpreBHV1 and the other for CpreGHV1 (Table 3).
In this study we identified one Sciurus variegatoides (sample \#10291) from a holding in Germany with a coinfection of VSBV-1 and SvarPyV1 and four Callosciurus prevostii (samples \#10295, \#10296, \#10303 and \#10304) from German holdings that were infected with VSBV-1 and CprePyV1.

\section{Amplification of complete polyomavirus genomes}

Complete genomes of ScarPyV1, CeryPyV1, and CprePyV1 were generated by nested LD-PCR using specific tail-to-tail primers (Fig. 1, Additional file 1 and Additional file 2). The LD-PCR products of around $5.2 \mathrm{kbp}$ were sequenced by classical primer-walking (primers not listed) and sequences of the (i) initial generic PCR products, (ii) LD-PCR products, and (iii) specific PCR fragments mentioned above were assembled to final circular genome sequences (Fig. 1). Full PyV genomes or partial $\mathrm{PyV}$ sequences are listed with countries of origin and GenBank accession numbers in Table 4.

ORF analysis with Geneious 11.1.5. software revealed the typical PyV genome organization with CDS for the viral capsid proteins VP1, VP2 and VP3, the regulatory proteins STAg and LTAg (but not middle $\mathrm{T}$ antigen (MTag)) on the opposite strand, and the NCCR (Fig. 1). As the LTAg CDS of all other mammalian PyVs and some of the STAg CDS are interrupted by an intron, we searched for such introns in the squirrel PyV genomes in silico and experimentally, as described in detail below. In addition, the VP2 ORF of CprePyV1 and ScarPyV1 was found to be interrupted by two (CprePyV1) or three (ScarPyV1) stop codons (not shown). Therefore, splicing of the VP2-encoding late mRNA was predicted and analyzed experimentally in cell culture. In upstream direction of the VP2 coding sequence ScarPyV1 and CprePyV1 display an additional ORF in their genome. In a few PyVs of the genus Betapolyomavirus, e.g. SV40 and BK virus, the so-called agnoprotein is encoded at this position. Agnoprotein was identified as a regulatory protein required for efficient virus proliferation. However, the proteins putatively encoded by ScarPyV1 and CprePyV1 do not show similarities with the known agnoproteins, and their function is currently unknown.

\section{Identification of splice sites in early and late regions of the novel polyomaviruses}

Splice donor and acceptor sites with high Human Splicing Finder (HSF) rating (75-95) and conserved in sequence and position compared to related PyVs were identified in LTAg CDS of all three squirrel PyVs. In addition, splice sites were predicted for STAg mRNA of CprePyV1, with the splice donor site interrupting the stop codon. The introns of STAg mRNA of ScarPyV1 and CeryPyV1 were found to be located after the stop codon. Finally, an intron was predicted for the VP2 CDS 


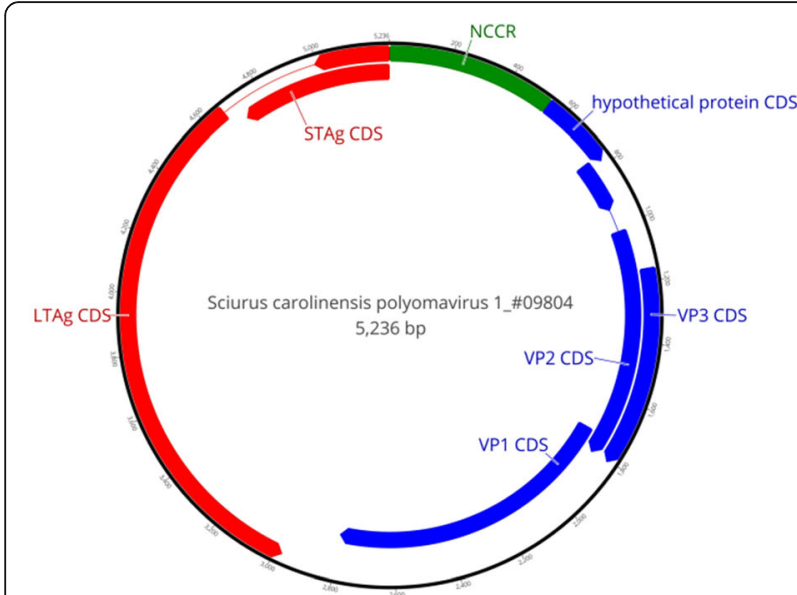

a

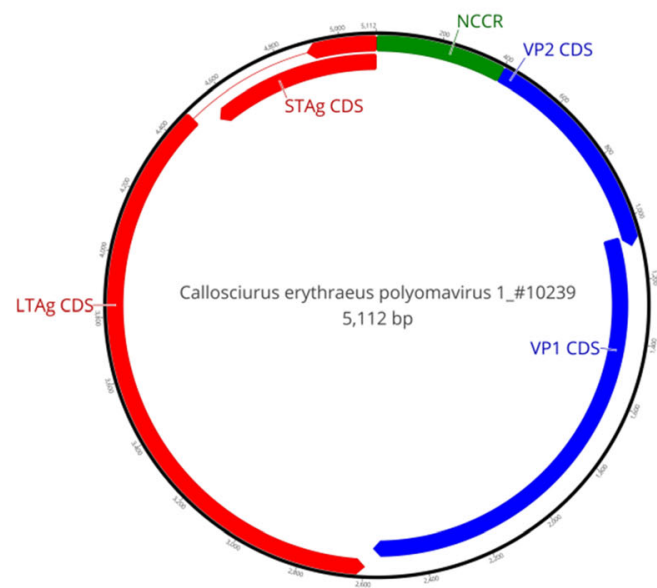

C

Fig. 1 Genome organization of Sciurus carolinensis PyV1 (a), Callosciurus prevostii PyV1 (b) and Callosciurus erythraeus PyV1 (c). Putative coding regions for VP1, VP2 and VP3 are marked by blue bars; putative STAg antigen and LTAg antigen coding regions are marked by red bars, each with arrow head indicating direction of transcription. Thin lines demarcate introns. Non-coding control region (NCCR) is marked by green bars. An additional hypothetical protein CDS was predicted in the genomes of ScarPyV1 and CprePyV1

of CprePyV1 and ScarPyV1 explaining the occurrence of an interrupted CDS (Fig. 2 and Table 5).

Experimental confirmation of the splice sites predicted for ScarPyV1 was performed with an approach that was used previously for splice site analysis of two human PyVs [54]. First we transfected either the early or late region with flanking sequences of ScarPyV1 (from sample \#9804) into monkey Vero cells and murine NIH/3T3 cells, isolated mRNA at the day of transfection and at days $1,2.5$ and 6 after transfection, and synthesized cDNA. Thereafter, a nested PCR was performed with primers that flank the putative introns (Additional file 1). By sequencing of the PCR products, the splice sites predicted for both the early (STAg and LTAg CDS) and the late region (VP2 CDS) were confirmed in Vero and NIH/ $3 \mathrm{~T} 3$ cells as described in detail below.

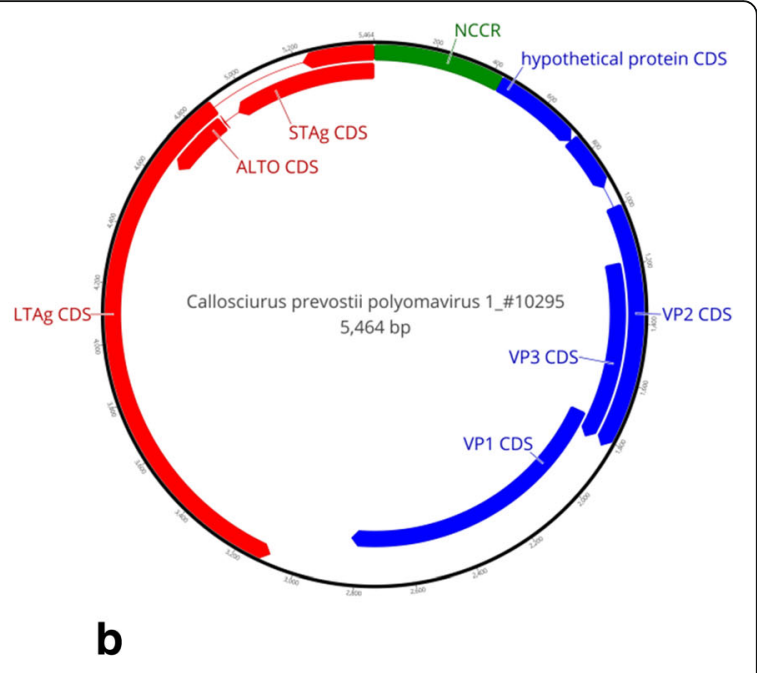

With two nested PCRs that respectively span $300 \mathrm{bp}$ and $500 \mathrm{bp}$ in the early region around the predicted STAg intron, a spliced mRNA was detected in Vero and NIH/3T3 cells that displays an unspliced CDS of $519 \mathrm{bp}$ and encodes the predicted STAg of 172 amino acids (aa). Behind the stop codon (nucleotide (nt) 517-519), a short intron of $72 \mathrm{nt}$ was observed (Fig. 2a and Table 5). With PCR that spans $800 \mathrm{bp}$ in the early region around the predicted LTAg intron, a spliced mRNA was detected in Vero and NIH/3T3 cells from which a spliced CDS of $1.935 \mathrm{kbp}$ (exon 1 and 2 in frame +1 ) was inferred. It encodes an LTAg of 644 aa (Table 5 and Fig. 2a). The ScarPyV1-encoded TAgs share the $81 \mathrm{~N}$ terminal aa.

Finally, we performed a PCR that spans $400 \mathrm{bp}$ in the late region around the predicted VP2 intron. A spliced 
Table 4 Novel polyoma- and herpesvirus sequences deposited in GenBank

\begin{tabular}{|c|c|c|c|c|c|}
\hline Virus name & $\begin{array}{l}\text { Country of } \\
\text { origin }\end{array}$ & Sample ID & Sequence length (bp) & $\begin{array}{l}\text { Complete } \\
\text { genome }\end{array}$ & GenBank accession number \\
\hline CeryPyV1 & Italy & $\# 10239, \# 10271, \# 10275$ & 5112 & + & MK671087, MK671088, MK671089 \\
\hline CprePyV1 & Germany $^{\mathrm{a}}$ & \#10295, \#10296, \#10304 & 5464 & + & MK883808, MK883809, MK883810 \\
\hline ScarPyV1 & UK & \#9804, \#9982, \#10018 & $5236,5237,5237$ & + & MK671101, MK671096, MK671097 \\
\hline SvarPyV1 & Germany $^{a}$ & \#10291 & 213 & & MK671090 \\
\hline CeryBHV1 & Italy & $\# 10257, \# 10259, \# 10262$ & 478 & & MK957142, MK957143, MK957144 \\
\hline CpreBHV1 & Germany $^{a}$ & \#10298 & 478 & & MN037512 \\
\hline ScarBHV1 & Italy & \#10197 & 412 & & MN047451 \\
\hline \multirow[t]{2}{*}{ SvulBHV1 } & UK & \#9807, \#9808, \#9813, \#9824 & $3336,3336,3327,3336$ & & $\begin{array}{l}\text { MK671091, MK671092, MK671093, } \\
\text { MK671094 }\end{array}$ \\
\hline & Germany & \#9886 & 3442 & & MK671095 \\
\hline CeryGHV1 & Italy & $\# 10276$ & 472 & & MK957139 \\
\hline CpreGHV1 & Germany $^{a}$ & $\# 10305$ & 166 & & not archivable in GenBank (<200 bp) \\
\hline ScarGHV1 & UK & \#9783, \#9800, \#9802 & 3334 & & MK671098, MK671099, MK671100 \\
\hline ScarGHV2 & USA & $\# 10179$ & 166 & & not archivable in GenBank (<200 bp) \\
\hline UricGHV1 & Canada & $\begin{array}{l}\# 10168, \# 10170, \# 10171, \# 10173, \\
\# 10174, \# 10175\end{array}$ & 3212 & & $\begin{array}{l}\text { MK671102, MK671 103, MK6711104, } \\
\text { MK671105, MK671106, MK671107 }\end{array}$ \\
\hline TstrGHV1 & USA & $\# 10182, \# 10183$ & 443 & & MK957140, MK957141 \\
\hline
\end{tabular}

animals originate from holdings

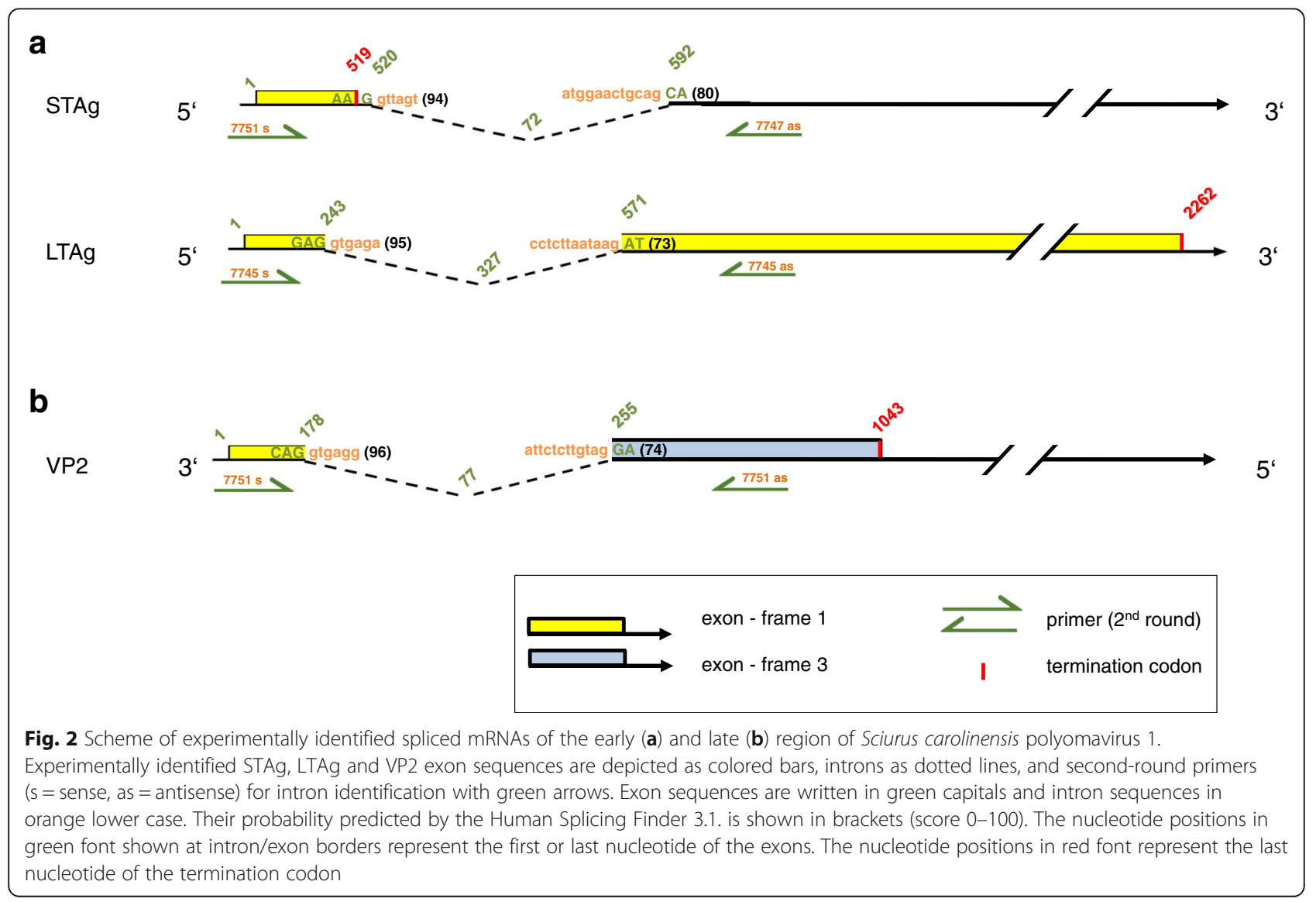


Table 5 Splice donor and acceptor sites in the early and late regions of the novel polyomaviruses

\begin{tabular}{|c|c|c|c|}
\hline Polyomavirus & $\mathrm{CDS}^{\mathrm{a}}$ & Splice donor site EXON|intron ${ }^{b}$ & $\begin{array}{l}\text { Splice acceptor site } \\
\text { intron|EXON }\end{array}$ \\
\hline \multirow[t]{3}{*}{ Sciurus carolinensis polyomavirus 1} & STAg & AAG|gttagt (94) & atggaactgcag $\mid C A(80)$ \\
\hline & LTAg & GAG|gtgaga (95) & cctcttaataag|AT (73) \\
\hline & VP2 & CAG|gtgagg (96) & attctcttgtag|GA (85) \\
\hline \multirow[t]{3}{*}{ Callosciurus prevostii polyomavirus 1} & STAg & ATG|gtgagt (93) & tacctttaacag|AT (85) \\
\hline & LTAg & GAG|gtaaaa (83) & tacctttaacag|AT (85) \\
\hline & VP2 & GAG|gtaaga (96) & ttccttttgtag|GA (87) \\
\hline Callosciurus erythraeus polyomavirus 1 & LTAg & GAG|gtacgt (89) & $\operatorname{tgcttctttcag|GA~(93)~}$ \\
\hline
\end{tabular}

${ }^{\mathrm{a}} \mathrm{CDS}$ coding sequence

${ }^{\mathrm{b}}$ score (0-100; in brackets) of similarity to the splice consensus site generated by the Human Splicing Finder 3.1

CDS was amplified from cDNA that displays two exons (Table 5 and Fig. 2b).

The experimental results generated for ScarPyV1 were completely in line with the theoretical predictions, i.e., all three predicted introns were detected in both tested cell lines and at different time points of harvesting. The splice sites predicted for LTAg, STAg, and VP2 CDS of CprePyV1 (Table 5) were not studied experimentally because the CprePyV1 genome is closely related to that of ScarPyV1 (see phylogenetic tree analysis below) and the splice sites of both PyVs are conserved in position and sequence. Likewise, splice sites predicted for CeryPyV1 were not studied experimentally as they are conserved in position and sequence with those of the closely related Philantomba monticola PyV1, whose sites were experimentally confirmed earlier [65].

\section{Determination of glycoprotein B and extended DNA polymerase nucleotide sequences of herpesviruses}

To extend the DPOL gene sequence information for each discovered HV in upstream direction into the adjacent $\mathrm{gB}$ CDS, all samples HV-positive in the DPOL PCR were reevaluated with generic PCRs that were reported earlier $[44,51,52,66]$ to target the gB sequences of either BHVs or GHVs (Additional file 1 and Additional file 3). These generic gB PCRs were less sensitive compared to the generic DPOL PCR, as we amplified a partial $g B$ sequence of ScarGHV1 from only one lung sample of 20 spleen and seven lung samples that had been positive in DPOL PCR. SvulBHV1 gB sequence was amplified from two spleen and three lung samples of 39 DPOL PCR-positive samples and UricGHV1 $\mathrm{gB}$ sequence was amplified from all 10 DPOL PCR-positive spleen samples. Next, we closed the sequence gap between the $\mathrm{gB}$ and the DPOL sequence (approximately $3.2 \mathrm{kbp}$ ) for each of the three HVs with LD-PCR and sequenced the gB-to-DPOL product by classical primer walking. This led to five continuous SvulBHV1 sequences with a length of $3.327-3.442 \mathrm{kbp}$, three ScarGHV1 sequences of $3.334 \mathrm{kbp}$, and five UricGHV1 sequences of $3.212 \mathrm{kbp}$ (Table 4).
For ScarBHV1, ScarGHV2, TstrGHV1, CpreBHV1, CpreGHV1, CeryBHV1 and CeryGHV1 the generic gB $\mathrm{PCR}$ did not produce the desired $\mathrm{gB}$ fragment. Therefore we extended the short DPOL sequences from around $170 \mathrm{bp}$ to $>400 \mathrm{bp}$ by using the outer sense primer of the generic DPOL PCR (285-S DFA; Additional file 1) in combination with two virus-specific antisense primers (Additional file 1) in hemi-nested PCR format. This approach was successful for ScarBHV1 (412 bp; from one sample), CeryBHV1 (478 bp; from three samples), CeryGHV1 (472 bp from one sample), CpreBHV1 (478 bp; from one sample) and TstrGHV-1 (443 bp from two samples), and failed for CpreGHV1 and ScarGHV2. The partial HV sequences are listed with countries of origin and GenBank accession numbers in Table 4.

\section{Phylogenetic analysis of conserved amino acid blocks of the polyomavirus LTAg and VP1 sequences}

For evolutionary conclusions and taxonomical classification phylogenetic analyses were done. The ML and MCC trees based on PyV LTAg aa sequences (Fig. 3 and Additional file 4) are quite similar and allow the following conclusions: the novel PyVs can be tentatively assigned to different genera within the family Polyomaviridae, as ScarPyV1 and CprePyV1 nest within the diversity of genus Betapolyomavirus and CeryPyV1 in the genus Alphapolyomavirus. Within the betapolyomaviruses the three ScarPyV1 (GenBank Accession numbers MK671096, MK671097 and MK671101) and the three CprePyV1 (GenBank Accession numbers MK883808 MK883810) cluster together in a well-supported monophyletic group, which also comprises another rodent polyomavirus, Glis glis polyomavirus 1 (GgliPyV1, GenBank Accession number MG701352), and Delphinus delphis polyomavirus 1 (DdelPyV1, GenBank Accession number KC594077). The close evolutionary relationship of these viruses is strengthened by the observation that their VP2 CDS is interrupted by an intron. This splicing event within the VP2 CDS has been either experimentally verified 


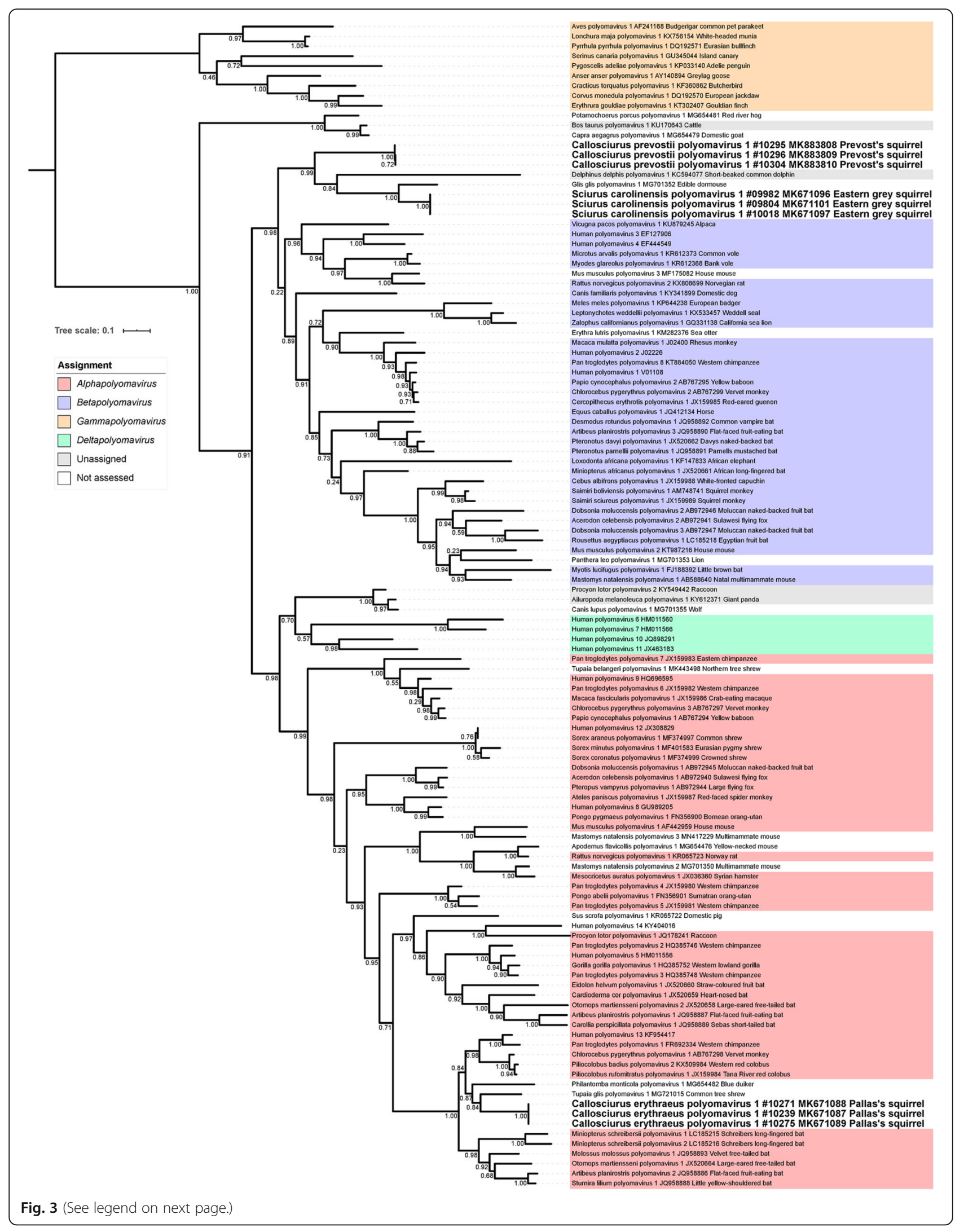


(See figure on previous page.)

Fig. 3 Maximum-likelihood (ML) tree analysis of polyomaviruses based on conserved amino acid blocks of the polyomavirus LTAg sequences. Phylogenetic relationships of polyomaviruses, including classification of the novel viruses, based on conserved amino acid blocks of LTAg sequence. Polyomaviruses are denoted by Latin taxonomic names of their hosts, GenBank accession number, common name of the species and - in case of the new viruses - sample ID. For International Committee on Taxonomy of Viruses (ICTV)-recognized species, virus genera are indicated by colors. Viruses newly identified in this study are given in bold font. Branch support was assessed using Shimodaira-Hasagawa-like approximate likelihood ratio tests (SH-like aLRT)

(Sciurus carolinensis polyomavirus 1 (this study) and Glis glis polyomavirus 1 [65]) or predicted in silico for Delphinus delphis polyomavirus 1, and Callosciurus prevostii polyomavirus 1 as the VP2 CDS of all clade members comprise highly conserved splice donor and acceptor motifs with high score $(>75)$ in the Human Splicing Finder 3.1.

All three representatives of the novel CeryPyV1 cluster together within the genus Alphapolyomavirus. Although their exact phylogenetic placement is uncertain, they belong to a well-supported clade of polyomaviruses infecting hosts of the orders Artiodactyla, Chiroptera, Primates and Scandentia (Fig. 3 and Additional file 4).

As expected and reported many times, the VP1-based ML and MCC trees (Additional file 5 and Additional file 6) support clades that differ from those delineating genera in the LTAg-based analyses [41]. In these trees, ScarPyV1 and CprePyV1 also formed a weakly supported monophyletic group with GgliPyV1 and DdelPyV1 but this group also included Canis familiaris polyomavirus 1 (GenBank Accession number KY341899). This virus may also have a spliced VP2 as it shares conserved VP2 splice sites with the other four polyomaviruses. The evolutionary position of SvarPyV1 (from the variegated squirrel) had to be allocated in the phylogenetic trees based on VP1 (Additional file 5 and Additional file 6), because only a partial VP1 sequence was identified. The single SvarPyV1 sequence clusters within the genus Alphapolyomavirus and is a sister virus to a PyV group consisting of an organ-utan and two chimpanzee PyVs. This phylogenetic allocation and the tentative host association of SvarPyV1 will need confirmation, once additional SvarPyV1 sequences from several animals and a complete SvarPyV1 LTAg sequence are available.

\section{Phylogenetic analysis of conserved amino acid blocks of herpesvirus partial DPOL and $\mathrm{gB}$ sequences}

The ML and MCC trees based on partial DPOL sequences of BHVs (Fig. 4 and Additional file 7) are very similar and show that the four identified squirrel BHVs form a separate cluster that is associated with moderate support to a clade comprising rodent HVs of the genus Muromegalovirus and other currently unclassified rodent HVs. The ML and MCC trees based on partial gB (Additional file 8 and Additional file 9) are very similar

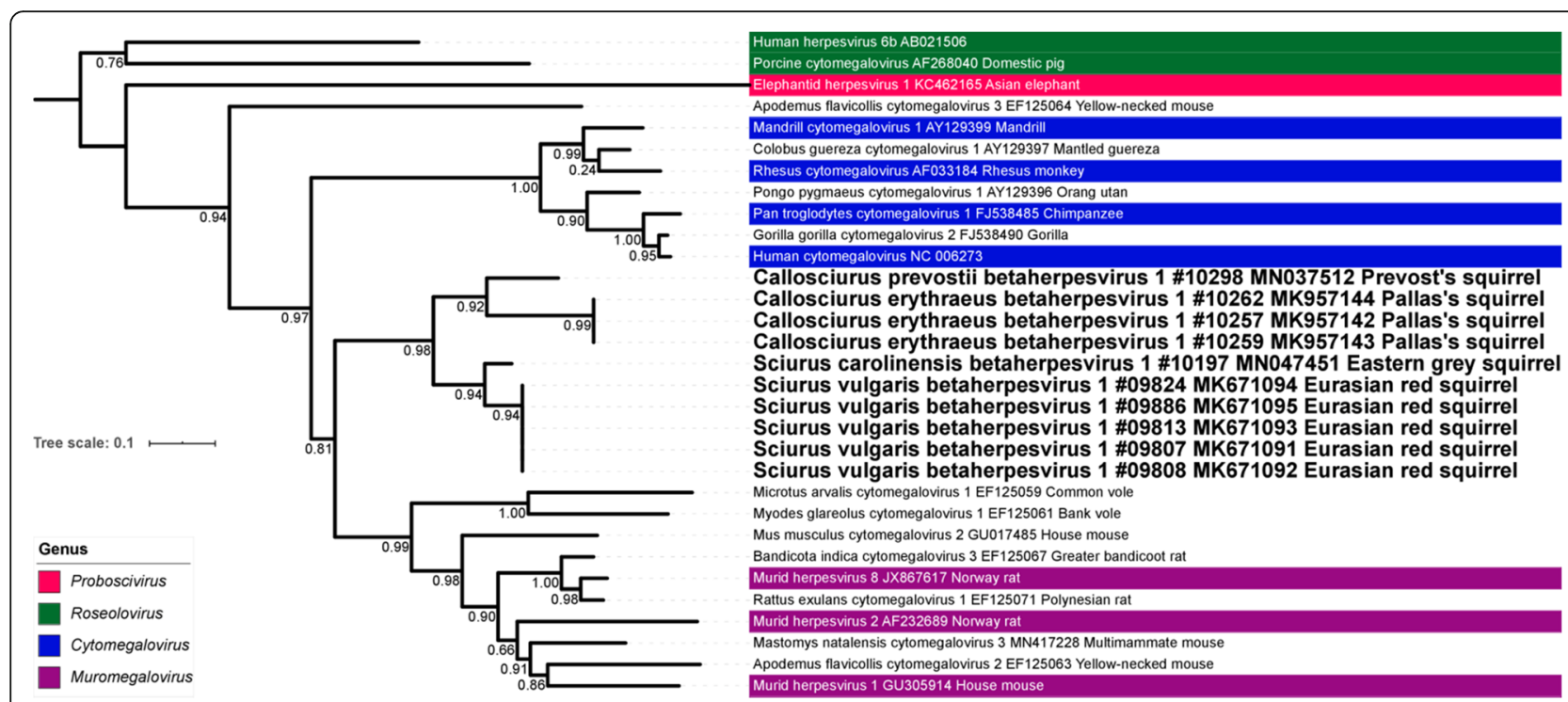

Fig. 4 Maximum-likelihood (ML) tree analysis of betaherpesviruses based on conserved amino acid blocks of the DPOL sequences. Phylogenetic relationships of betaherpesviruses, including classification of the novel viruses, based on conserved amino acid blocks of DPOL sequence. Betaherpesviruses are denoted by Latin taxonomic names or common names of their hosts, GenBank accession number, common name of the species and - in case of the new viruses - sample ID. For ICTV-recognized species, virus genera are indicated by colors. Viruses newly identified in this study are given in bold font. Branch support was assessed using Shimodaira-Hasagawa-like approximate likelihood ratio tests (SH-like aLRT) 
to the DPOL-based trees but only the Sciurus vulgaris betaherpesvirus 1 sequences are included as for the other three BHVs gB sequences were not available.

The ML and MCC phylogenetic trees based on partial DPOL (Fig. 5 and Additional file 10) and partial gB of GHVs (Additional file 11 and Additional file 12) are also quite similar and show that the squirrel GHVs form two separate groups. One group nests within GHVs of the genus Rhadinovirus and forms a weakly supported clade with a group of rodent HVs (in all trees) and a rhadinovirus of the South American tapir (only in DPOL-based trees). The other squirrel HV group nests within a clade that comprises ungulate GHVs of the genus Macavirus, a GHV of African elephant and the human Epstein-Barr virus (species Human herpes virus 4).

\section{Discussion}

The screening of more than 200 squirrels from holdings and sampled wildlife, either indigenous or introduced species, from five countries on two continents resulted in the discovery of four novel PyVs, four novel BHVs and six novel GHVs. Phylogenetic analysis allocated the novel squirrel PyVs to the genera Alphapolyomavirus and Betapolyomavirus, the novel BHVs to the herpesvirus genus Muromegalovirus, and the GHVs to the genera Rhadinovirus and Macavirus. This first description of PyVs, BHVs and GHVs in squirrels of different species increased our knowledge (e.g. [51, 65] on the diversity of PyVs and HVs in rodents. In particular, some of these viruses represent novel highly divergent lineages or sublineages in the corresponding phylogenetic trees.

The results of our PyV and HV investigations furthermore show that (i) the virus-specific nested PCRs have a higher sensitivity than the generic PCRs, thereby increasing the number of PyV-and HV-positive samples and positive individuals and (ii) virus detection was more frequent in spleen, compared to lung samples. The adapted methods were used here for a first prevalence estimation for some of these viruses, allowing an initial comparison of the prevalences in indigenous and introduced populations.
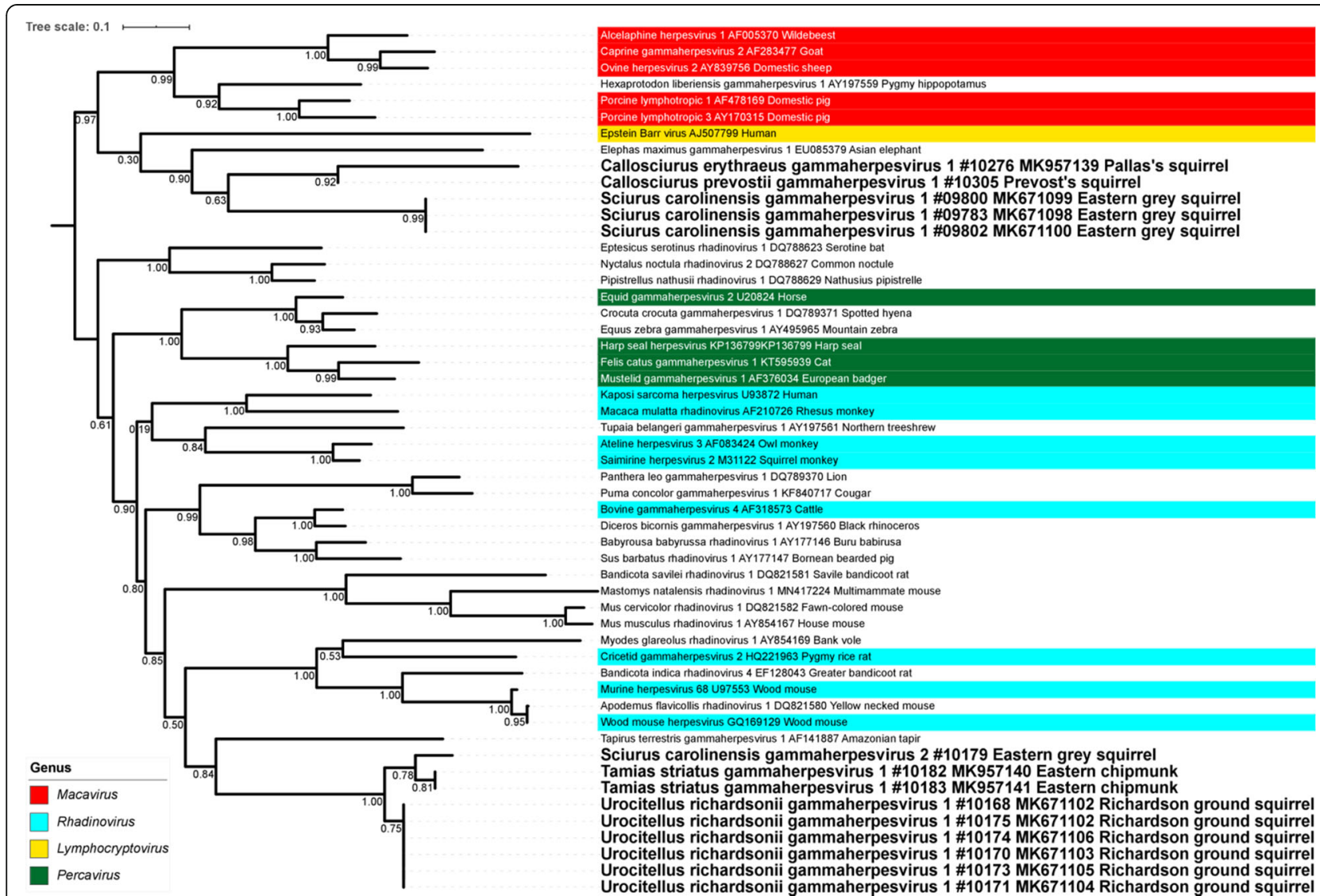

Fig. 5 Maximum-likelihood (ML) tree analysis of gammaherpesviruses based on conserved amino acid blocks of the DPOL sequences. Phylogenetic relationships of gammaherpesviruses, including classification of the novel viruses, based on conserved amino acid blocks of DPOL sequence. Gammaherpesviruses are denoted by Latin taxonomic names or common names of their hosts, GenBank accession number, common name of the species and - in case of the new viruses - sample ID. For ICTV-recognized species, virus genera are indicated by colors. Viruses newly identified in this study are given in bold font. Branch support was assessed using Shimodaira-Hasagawa-like approximate likelihood ratio tests (SH-like aLRT) 
Based on the prevalence of the different viruses and their detection in exclusively only one respective squirrel species, it is likely that the viruses are all species-specific and were detected in their respective natural hosts. The fact that the novel squirrel PyVs and HVs or closely related ones were not detected previously in other host species may further strengthen this assumption of host specificity. These investigations thereby improve our knowledge on the host specificity of PyVs and HVs [29, 41].

Our study indicated a broad geographical distribution of some of the novel viruses: ScarPyV1 was detected in the Eastern Grey squirrel from the original range in North America, but also in introduced populations in Great Britain and Italy. Similarly, both ScarGHV1 and ScarGHV2 were detected in the original North American and the introduced British populations. The detection of SvulBHV1 in Eurasian red squirrels from Germany and Scotland might be explained by the long, interlinked history of Eurasian red squirrels on the British Isles with other European populations as discussed previously for the detection of Eurasian red squirrel-associated Squirrel adenovirus 1 (SqAdV-1) strains of high sequence similarity in Germany and Scotland [13].

For three PyVs complete genomes were generated and splice sites were experimentally determined for one of these novel viruses. As reported earlier [65], such experimental determination is critically important for annotation of coding PyV sequences. The search for the reservoir of VSBV-1 within this study resulted in solely negative findings, although additional squirrel species were investigated. This confirms results of our previous study that the Eastern grey squirrel is most likely not the reservoir of bornaviruses [21] and suggests that the Pallas's squirrel (Callosciurus erythraeus) is also not a reservoir host for known bornaviruses, at least in the investigated introduced Italian population. It is still unclear and requires further investigation why squirrels of two different subfamilies in German private and zoo holdings which were imported from different geographic origins (Sciurus variegatoides and Callosciurus prevostii) harbor VSBV-1 sequences [21] of such high similarity. Further screening approaches, including squirrels and other small mammals, will focus on the identification of other possible reservoir hosts of orthobornaviruses in the future. Thereby, it should be evaluated if (i) VSBV-1 was imported with squirrels from Central America or South East Asia and afterwards spread in the German squirrel holdings and holdings in The Netherlands and Croatia, or if (ii) another, yet unknown reservoir host of VSBV-1 exists in Central Europe. Furthermore, experimental infection studies will be done to evaluate the VSBV-1 susceptibility of different squirrel species.
In this study we identified SvarPyV1 in a Sciurus variegatoides from a German holding that was tested positive for VSBV-1 in a previous study and CprePyV1 in four Callosciurus prevostii from German holdings that were also tested VSBV-1-positive before [17, 21, 22]. These observations indicate viral coinfections in squirrels, confirming results from previous studies in other rodents [67-69]. Evidence for bi-directional interplay of viral and/or bacterial agents, e.g. altering the host's susceptibility, the disease progression, severity of the disease and the host's immune response in rodents have been reported [70,71]. However, it is currently unknown if the agents investigated in the current study affect each other, and we have no direct evidence if the detected agents are causing clinical signs or pathologic alterations in the investigated squirrels.

\section{Conclusions}

This is the first report on molecular identification and sequence characterization of PyVs and HVs in rodents of the family Sciuridae. These findings will allow further targeted screenings of squirrels of the investigated species to analyze the role that these novel viruses play on the population dynamics and competitive interactions in wildlife squirrel populations. Furthermore, the origin of these novel viruses and their spatially and temporally driven evolution in indigenous and introduced populations of grey squirrels, Pallas's squirrels and Prevost's squirrels and questions regarding the interactions of different agents in squirrels are interesting topics for future investigations.

\section{Supplementary information}

Supplementary information accompanies this paper at https://doi.org/10. 1186/s12985-020-01310-4.

Additional file 1. PCR assays for detection of borna-, polyoma- and herpesviruses and mycoplasma.

Additional file 2. Flow chart of multi-level PCR analysis for detection of squirrel polyomaviruses. Generic nested VP1 PCR (second-round product displayed as magenta-coloured bar) with degenerate primers was conducted. For full genome amplification, this was followed by specific nested long-distance PCR (LD-PCR; second-round product of approximately 5 kbp shown as red bar) and overlapping standard nested PCR (second-round product of approximately $800 \mathrm{bp}$ shown as green bar) with specific primers. Grey bars represent coding sequences, black bar the non-coding control region.

Additional file 3. Flow chart of multi-level PCR analysis for detection of squirrel herpesviruses. Generic nested DPOL PCR (product: bar in magenta) with degenerate primers was carried out. For extended sequence determination, this was followed by generic gB PCR (blue) with degenerate primers and subsequent long-distance PCR (LD-PCR) (red) with specific primers. Products of the second PCR rounds are shown. The sequences of the generic DPOL PCR product and the extended DPOL PCR product build a contiguous sequence of $0.4-0.5 \mathrm{kbp}$ (black). The sequences of the generic $\mathrm{gB}$ and the generic DPOL PCR product build together with the LD-PCR-derived sequence a contiguous sequence of approximately $3.3 \mathrm{kbp}$ (black). On top of the figure, coding sequences are displayed by grey bars. The arrow heads indicate the direction of transcription. 
Additional file 4. Maximum clade credibility tree analysis of polyomaviruses based on conserved amino acid blocks of the LTAg sequences. Phylogenetic relationships of polyomaviruses, including classification of the novel viruses, based on conserved amino acid blocks of LTAg sequence. Branch support values displayed at the nodes correspond to their posterior probability. For further details see legend of Fig. 3.

Additional file 5. Maximum likelihood tree analysis of polyomaviruses based on conserved amino acid blocks of the VP1 sequences. Phylogenetic relationships of polyomaviruses, including classification of the novel viruses, based on conserved amino acid blocks of VP1 sequence. Branch support values displayed at the nodes were assessed using Shimodaira-Hasagawa-like approximate likelihood ratio tests ( $\mathrm{SH}$ like aLRT). For further details see legend of Fig. 3.

Additional file 6. Maximum clade credibility tree analysis of polyomaviruses based on conserved amino acid blocks of the VP1 sequences. Phylogenetic relationships of polyomaviruses, including classification of the novel viruses, based on conserved amino acid blocks of VP1 sequence. Branch support values displayed at the nodes correspond to their posterior probability. For further details see legend of Fig. 3.

Additional file 7. Maximum clade credibility tree analysis of betaherpesviruses based on conserved amino acid blocks of the DPOL sequences. Phylogenetic relationships of betaherpesviruses, including classification of the novel viruses, based on conserved amino acid blocks of DPOL sequence. Branch support values displayed at the nodes correspond to their posterior probability. For further explanation see legend of Fig. 4.

Additional file 8. Maximum likelihood tree analysis of betaherpesviruses based on conserved amino acid blocks of the gB sequences. Phylogenetic relationships of betaherpesviruses, including classification of the novel viruses, based on conserved amino acid blocks of $\mathrm{gB}$ sequence. Branch support values displayed at the nodes were assessed using Shimodaira-Hasagawa-like approximate likelihood ratio tests ( $\mathrm{SH}$-like aLRT). For further explanation see legend of Fig. 4.

Additional file 9. Maximum clade credibility tree analysis of betaherpesviruses based on conserved amino acid blocks of the $\mathrm{gB}$ sequences. Phylogenetic relationships of betaherpesviruses, including classification of the novel viruses, based on conserved amino acid blocks of $\mathrm{gB}$ sequence. Branch support values displayed at the nodes correspond to their posterior probability. For further explanation see legend of Fig. 4.

Additional file 10. Maximum clade credibility tree analysis of gammaherpesviruses based on conserved amino acid blocks of the DPOL sequences. Phylogenetic relationships of gammaherpesviruses, including classification of the novel viruses, based on conserved amino acid blocks of DPOL sequence. Branch support values displayed at the nodes correspond to their posterior probability. For further explanation see legend of Fig. 5 .

Additional file 11. Maximum likelihood tree analysis of gammaherpesviruses based on conserved amino acid blocks of the $\mathrm{gB}$ sequences. Phylogenetic relationships of gammaherpesviruses, including classification of the novel viruses, based on conserved amino acid blocks of $\mathrm{gB}$ sequence. Branch support values displayed at the nodes were assessed using Shimodaira-Hasagawa-like approximate likelihood ratio tests (SH-like aLRT). For further explanation see legend of Fig. 5.

Additional file 12. Maximum clade credibility tree analysis of gammaherpesviruses based on conserved amino acid blocks of the $\mathrm{gB}$ sequences. Phylogenetic relationships of gammaherpesviruses, including classification of the novel viruses, based on conserved amino acid blocks of $\mathrm{gB}$ sequence. Branch support values displayed at the nodes correspond to their posterior probability. For further explanation see legend of Fig. 5.

\section{Abbreviations}

aa: Amino acid; as: Antisense; BHVs: Betaherpesviruses; BLASTn: Nucleotide Basic Local Alignment Search Tool; BMCMC: Bayesian Markov chain Monte Carlo; BoDV-1: Borna Disease Virus 1; BoDV-2: Borna Disease Virus 2; bp: Base pairs; CDS: Coding sequence; CeryBHV1: Callosciurus erythraeus betaherpesvirus 1; CeryGHV1: Callosciurus erythraeus gammaherpesvirus 1; CeryPyV1: Callosciurus erythraeus polyomavirus 1; CpreBHV1: Callosciurus prevostii betaherpesvirus 1; CpreGHV1: Callosciurus prevostii gammaherpesvirus 1; CprePyV1: Callosciurus prevostii polyomavirus 1; DdelPyV1: Delphinus delphis polyomavirus 1; DMEM: Dulbecco's minimal Eagle medium; DMSO: Dimethyl sulfoxide; dNTP: Dideoxynucleoside triphosphate; DPOL: DNA polymerase; ECACC: European Collection of Authenticated Cell Cultures; FCS: Fetal calf serum; gB: Glycoprotein B; GgliPyV1: Glis glis polyomavirus 1; GHVs: Gammaherpesviruses; HSF: Human Splicing Finder; HVs: Herpesviruses; ICTV: International Committee on Taxonomy of Viruses; kb: Kilobases; kbp: Kilobase pairs; LD-PCR: Longdistance PCR; LTAg: Large T-antigen; MCC: Maximum clade credibility; MCPyV: Merkel cell polyomavirus; ML: Maximum likelihood; MTAg: Middle Tantigen; NCCR: Non-coding control region; nt: Nucleotide; ORF: Open reading frame; PCR: Polymerase chain reaction; PhyML-SMS: PhyML V3 with smart model selection; PST: Posterior set of trees; PyVs: Polyomaviruses; RTqPCR: Reverse transcription-quantitative polymerase chain reaction; s: Sense; ScarBHV1: Sciurus carolinensis betaherpesvirus 1; ScarGHV1: Sciurus carolinensis gammaherpesvirus 1; ScarGHV2: Sciurus carolinensis gammaherpesvirus 2; ScarPyV1: Sciurus carolinensis polyomavirus 1; SH-like aLRT: ShimodairaHasegawa-like approximate likelihood ratio test; SqAdV-1: Squirrel adenovirus 1; STAg: Small T-antigen; SvarPyV1: Sciurus variegatoides polyomavirus 1; SvulBHV1: Sciurus vulgaris betaherpesvirus 1; TstrGHV1: Tamias striatus gammaherpesvirus 1; UricGHV1: Urocitellus richardsonii gammaherpesvirus 1; VSBV-1: Variegated squirrel bornavirus 1

\section{Acknowledgements}

The supply of Eurasian red squirrel samples from Germany by Tanya Lenn, Korinna Seybold, Jessica Hoch, Sabine Gallenberger, Tanja Schäfer, Stefan Bosch, and Herrmann Ansorge is gratefully acknowledged. We also thank the Second Changes Wildlife Rehabilitation Center, the Carbon County Environmental Education Center, the Pocono Wildlife Rehabilitation and Education Center, Morgan McIntyre, Timothy Lavoie, Kayla Eller, Ryan Giberson and Samantha Verespy for assistance with squirrel collection in the USA, Lucas Wauters with squirrel collection in Italy, as well as James F. Staples, James Hare, Colin Garroway, Lucy Johnson and Stephen Petersen for sending squirrel samples from Canada. The Scottish Wildlife Trust, Stephanie Johnstone, Ann-Marie MacMaster, Bryan Collins and Kirsty Kenny are kindly acknowledged for support in collecting British grey squirrel samples. Further thanks go to Jana Schulz for initial help with phylogenetic analyses, Anna Katarina Schilling, University of Edinburgh, Scotland for excellent support during dissections and Cornelia Walter and Melanie Fechtner, Robert KochInstitute, Berlin, for excellent technical assistance in the lab.

\section{Authors' contributions}

VS did data curation, formal analysis, sample acquisition, investigation of the samples for bornavirus, PyVs and HVs, visualization of the data and was the major contributor in writing the manuscript and editing. PWWL helped organizing samples in the UK, and supported writing the squirrel background part in the original draft and through review and editing. NF and CR provided samples from Italy and were part of the review and editing process. MAS and SM sent samples from the USA and helped review and editing. MVM was involved in the squirrel collection in Italy and supported through review and editing. SCS did the phylogenetic analyses and tree reconstruction. KS investigated squirrel samples for bornavirus belonging to previous studies, did formal analysis, validation of the data and review and editing. MB was responsible for funding acquisition, project administration, supervision and review and editing of the manuscript. RGU took part in the conceptualization, funding acquisition, project administration, supervision, data validation, writing the original draft and review and editing. BE contributed to the conceptualization, data curation, formal analysis, methodology, supervision, data validation, writing the original draft and supporting the review and editing process. The author(s) read and approved the final manuscript.

\section{Funding}

This work was funded in part by the Federal Ministry of Education and Research within the Research Network Zoonotic Infections to the 'Zoonotic Bornavirus Consortium' (ZooBoCo; grant no. 01KI1722A to MB and RGU). 


\section{Availability of data and materials}

All data generated or analyzed during this study are included in this published article.

Sequences of the novel viruses have been deposited in GenBank with accession numbers MK671087, MK671088, MK671089, MK671090, MK671091, MK671092, MK671093, MK671094, MK671095, MK671096, MK671097, MK671098, MK671099, MK671100, MK671101, MK671102, MK671103, MK671104, MK671105, MK671106, MK671107, MK883808, MK883809, MK883810, MK957139, MK957140, MK957141, MK957142, MK957143, MK957144, MN037512, MN047451.

\section{Ethics approval and consent to participate}

Animal carcasses investigated in this study were collected as part of population monitoring in the context of field research and were from animals that had been found dead, died at wildlife rescue centers, or had been humanely culled as part of invasive species or disease control efforts. No animals were directly sacrificed for the purpose of this study. Therefore, the need for ethical approval was not applicable. All relevant guidelines for the use and handling of animals in scientific studies were strictly followed.

\section{Consent for publication}

N/A

\section{Competing interests}

The authors declare that they have no competing interests.

\section{Author details}

${ }^{1}$ Institute of Novel and Emerging Infectious Diseases,

Friedrich-Loeffler-Institut, Greifswald - Insel Riems, Germany. ${ }^{2}$ Royal (Dick) School of Veterinary Studies and Roslin Institute, University of Edinburgh, Roslin, Scotland, UK. ${ }^{3}$ Department of Veterinary Medicine, Università degli Studi di Milano, Milan, Italy. ${ }^{4}$ Department of Biology, Wilkes University, Wilkes-Barre, PA, USA. ${ }^{5}$ Department of Theoretical and Applied Sciences, Università degli Studi dell'Insubria, Varese, Italy. "P3 "Viral Evolution", Robert Koch-Institute, Berlin, Germany. ${ }^{7}$ Institute of Diagnostic Virology, Friedrich-Loeffler-Institut, Greifswald-Insel Riems, Germany. ${ }^{8}$ German Center for Infection Research (DZIF), partner site Hamburg - Lübeck - Borstel Greifswald-Insel Riems, Greifswald-Insel Riems, Germany. ${ }^{9}$ Division 12 'Measles, Mumps, Rubella and Viruses Affecting Immunocompromised Patients', Robert Koch-Institute, Berlin, Germany.

\section{Received: 21 January 2020 Accepted: 28 February 2020} Published online: 27 March 2020

\section{References}

1. Thorington RW Jr, Koprowski JL, Steele MA, Whatton JF. Squirrels of the world. Baltimore: Johns Hopkins University Press; 2012.

2. Bosch S, Lurz PWW. The Eurasian red squirrel: Verlags KG Wolf (formerly Westarp Wissenschaften); 2012.

3. Lurz PWW, Gurnell J, Magris L. Sciurus vulgaris (Rodentia: Sciuridae). Mamm Species. 2005;769:1-10.

4. Koprowski JL. Sciurus carolinensis (Rodentia: Sciuridae). Mamm Species. 1994:480:1-9.

5. Peacock DE. The grey squirrel Sciurus carolinensis in Adelaide, South Australia: its introduction and eradication. Vic Nat. 2009;126(4):150-6.

6. Gurnell J, Wauters LA, Lurz PWW, Tosi G. Alien species and interspecific competition: effects of introduced eastern grey squirrels on red squirrel population dynamics. J Anim Ecol. 2004;73(1):26-35.

7. Wauters LA, Lurz PWW, Gurnell JJER. Interspecific effects of grey squirrels (Sciurus carolinensis) on the space use and population demography of red squirrels (Sciurus vulgaris) in conifer plantations. Ecol Res. 2000;15(3):271-84.

8. White A, Bell SS, Lurz PWW, Boots M. Conservation management within strongholds in the face of disease-mediated invasions: red and grey squirrels as a case study. J Appl Ecol. 2014;51(6):1631-42.

9. Sainsbury AW, Deaville R, Lawson B, Cooley WA, Farelly SS, Stack MJ, et al. Poxviral disease in red squirrels Sciurus vulgaris in the UK: spatial and temporal trends of an emerging threat. Ecohealth. 2008:5(3):305-16.

10. Avanzi C, Del-Pozo J, Benjak A, Stevenson K, Simpson VR, Busso P, et al. Red squirrels in the British Isles are infected with leprosy bacilli. Science. 2016; 354(6313):744-7.
11. Abendroth $B$, Höper $D$, Ulrich $R G$, Larres $G$, Beer $M$. A red squirrel associated adenovirus identified by a combined microarray and deep sequencing approach. Arch Virol. 2017;162(10):3167-72.

12. Romeo C, Ferrari N, Rossi C, Everest DJ, Grierson SS, Lanfranchi P, et al. Ljungan virus and an adenovirus in Italian squirrel populations. J Wildl Dis. 2014;50(2):409-11.

13. Wernike K, Wylezich C, Hoper D, Schneider J, Lurz PWW, Meredith A, et al. Widespread occurrence of squirrel adenovirus 1 in red and grey squirrels in Scotland detected by a novel real-time PCR assay. Virus Res. 2018;257:113-8.

14. Bertolino S, Lurz PWW. Callosciurus squirrels: worldwide introductions, ecological impacts and recommendations to prevent the establishment of new invasive populations. Mammal Rev. 2013;43(1):22-33.

15. Lurz PWW, Fielding I, Hayssen V. Callosciurus prevostii (Rodentia: Sciuridae). Mamm Species. 2017:49(945):40-50.

16. Lurz PWW, Hayssen V, Geissler K, Bertolino S. Callosciurus erythraeus (Rodentia: Sciuridae). Mamm Species. 2013;45(902):60-74.

17. Hoffmann B, Tappe D, Höper D, Herden C, Boldt A, Mawrin C, et al. A variegated squirrel bornavirus associated with fatal human encephalitis. $N$ Engl J Med. 2015;373(2):154-62.

18. Best TL. Sciurus variegatoides (Rodentia: Sciuridae). Mamm Species. 1995; 500:1-6.

19. Daszak $P$, Cunningham AA, Hyatt AD. Emerging infectious diseases of wildlife--threats to biodiversity and human health. Science. 2000;287(5452): 443-9.

20. Hulme PE. Invasive species challenge the global response to emerging diseases. Trends Parasitol. 2014;30(6):267-70.

21. Schlottau K, Hoffmann B, Homeier-Bachmann T, Fast C, Ulrich RG, Beer M, et al. Multiple detection of zoonotic variegated squirrel bornavirus 1 RNA in different squirrel species suggests a possible unknown origin for the virus. Arch Virol. 2017;162(9):2747-54

22. Schlottau K, Jenckel M, van den Brand J, Fast C, Herden C, Höper D, et al. Variegated squirrel bornavirus 1 in squirrels, Germany and the Netherlands. Emerg Infect Dis. 2017;23(3):477-81.

23. Tappe D, Schlottau K, Cadar D, Hoffmann B, Balke L, Bewig B, et al. Occupation-associated fatal limbic encephalitis caused by variegated squirrel bornavirus 1, Germany, 2013. Emerg Infect Dis. 2018;24(6):978-87.

24. Staeheli P, Sauder C, Hausmann J, Ehrensperger F, Schwemmle M. Epidemiology of borna disease virus. J Gen Virol. 2000;81(Pt 9):2123-35.

25. Coras R, Korn K, Kuerten S, Huttner HB, Ensser A. Severe bornavirusencephalitis presenting as Guillain-Barre-syndrome. Acta Neuropathol. 2019; 137:1017.

26. Korn K, Coras R, Bobinger T, Herzog SM, Lucking H, Stohr R, et al. Fatal encephalitis associated with borna disease virus 1. N Engl J Med. 2018; 379(14):1375-7.

27. Liesche F, Ruf V, Zoubaa S, Kaletka G, Rosati M, Rubbenstroth D, et al. The neuropathology of fatal encephalomyelitis in human borna virus infection. Acta Neuropathol. 2019;138:653.

28. Schlottau K, Forth L, Angstwurm K, Höper D, Zecher D, Liesche F, et al. Fatal encephalitic borna disease virus 1 in solid-organ transplant recipients. N Engl J Med. 2018;379(14):1377-9.

29. Davison AJ, Eberle R, Ehlers B, Hayward GS, McGeoch DJ, Minson AC, et al The order Herpesvirales. Arch Virol. 2009;154(1):171-7.

30. Gedvilaite A, Tryland M, Ulrich RG, Schneider J, Kurmauskaite V, Moens U, et al. Novel polyomaviruses in shrews (Soricidae) with close similarity to human polyomavirus 12. J Gen Virol. 2017;98(12):3060-7.

31. Gjoerup O, Chang Y. Update on human polyomaviruses and cancer. Adv Cancer Res. 2010;106:1-51.

32. Groenewoud MJ, Fagrouch Z, van Gessel S, Niphuis H, Bulavaite A, Warren $\mathrm{KS}$, et al. Characterization of novel polyomaviruses from Bornean and Sumatran orang-utans. J Gen Virol. 2010;91(Pt 3):653-8.

33. Van Borm S, Rosseel T, Behaeghel I, Saulmont M, Delooz L, Petitjean T, et al. Complete genome sequence of bovine polyomavirus type 1 from aborted cattle, isolated in Belgium in 2014. Genome Announc. 2016;4(2):e01646.

34. Dutta SK, Gorgacz EJ, Albert TF, Ingling AL. Isolation of a herpesvirus from the cell culture of a malignant melanoma of a ground squirrel (Spermophilus tridecemlineatus). Am J Vet Res. 1977;38(5):591-5.

35. Diosi P, Plavosin L, Arcan P, David C. Recovery of a new herpesvirus from the ground squirrel (Citellus citellus). Pathol Microbiol (Basel). 1975;42(1):42-8.

36. Barahona H, Daniel MD, Katz SL, Ingalls JK, Melendez LV, King NW. Isolation and in vitro characterization of a herpesvirus from ground squirrels (Citellus sp). Lab Anim Sci. 1975;25(6):735-40. 
37. Moens U, Krumbholz A, Ehlers B, Zell R, Johne R, Calvignac-Spencer S, et al. Biology, evolution, and medical importance of polyomaviruses: an update. Infect Genet Evol. 2017;54:18-38.

38. Spurgeon ME, Lambert PF. Merkel cell polyomavirus: a newly discovered human virus with oncogenic potential. Virology. 2013;435(1):118-30.

39. Feltkamp MC, Kazem S, van der Meijden E, Lauber C, Gorbalenya AE. From Stockholm to Malawi: recent developments in studying human polyomaviruses. J Gen Virol. 2013;94(Pt 3):482-96.

40. Moens U, Van Ghelue M, Johannessen M. Oncogenic potentials of the human polyomavirus regulatory proteins. Cell Mol Life Sci. 2007;64(13): 1656-78.

41. Calvignac-Spencer S, Feltkamp MC, Daugherty MD, Moens U, Ramqvist T, Johne $\mathrm{R}$, et al. A taxonomy update for the family Polyomaviridae. Arch Virol. 2016;161(6):1739-50

42. Roizman B, Pellett P, Knipe D, Howley P, Griffin D. The family Herpesviridae: a brief introduction. Fields Virol. 2001;4: Lippincott, William \& Wilkins:2381-97.

43. Ben Salem N, Leendertz FH, Ehlers B. Genome sequences of polyomaviruses from the wild-living red colobus (Piliocolobus badius) and Western Chimpanzee (Pan troglodytes verus). Genome Announc. 2016;4(5):e01101.

44. Chmielewicz B, Goltz M, Ehlers B. Detection and multigenic characterization of a novel gammaherpesvirus in goats. Virus Res. 2001;75(1):87-94.

45. Chmielewicz B, Goltz M, Lahrmann KH, Ehlers B. Approaching virus safety in xenotransplantation: a search for unrecognized herpesviruses in pigs. Xenotransplantation. 2003;10(4):349-56.

46. Ehlers B, Borchers K, Grund C, Frolich K, Ludwig H, Buhk HJ. Detection of new DNA polymerase genes of known and potentially novel herpesviruses by PCR with degenerate and deoxyinosine-substituted primers. Virus Genes. 1999;18(3):211-20

47. Korup S, Rietscher J, Calvignac-Spencer S, Trusch F, Hofmann J, Moens U, et al. Identification of a novel human polyomavirus in organs of the gastrointestinal tract. PLoS One. 2013;8(3):e58021.

48. Leendertz FH, Scuda N, Cameron KN, Kidega T, Zuberbuhler K, Leendertz SA, et al. African great apes are naturally infected with polyomaviruses closely related to Merkel cell polyomavirus. J Virol. 2011;85(2):916-24.

49. Scuda N, Hofmann J, Calvignac-Spencer S, Ruprecht K, Liman P, Kuhn J, et al. A novel human polyomavirus closely related to the African green monkey-derived lymphotropic polyomavirus. J Virol. 2011;85(9):4586-90.

50. Schlegel M, Ali HS, Stieger N, Groschup MH, Wolf R, Ulrich RG. Molecular identification of small mammal species using novel cytochrome $B$ genederived degenerated primers. Biochem Genet. 2012;50(5-6):440-7.

51. Ehlers B, Kuchler J, Yasmum N, Dural G, Voigt S, Schmidt-Chanasit J, et al. Identification of novel rodent herpesviruses, including the first gammaherpesvirus of Mus musculus. J Virol. 2007;81(15):8091-100.

52. Prepens S, Kreuzer KA, Leendertz F, Nitsche A, Ehlers B. Discovery of herpesviruses in multi-infected primates using locked nucleic acids (LNA) and a bigenic PCR approach. Virol J. 2007;4:84.

53. van Kuppeveld FJ, Johansson KE, Galama JM, Kissing J, Bolske G, van der Logt JT, et al. Detection of mycoplasma contamination in cell cultures by a mycoplasma group-specific PCR. Appl Environ Microbiol. 1994;60(1):149-52.

54. Korup-Schulz SV, Lucke C, Moens U, Schmuck R, Ehlers B. Large T antigen variants of human polyomaviruses 9 and 12 and seroreactivity against their N terminus. J Gen Virol. 2017;98(4):704-14

55. Kearse M, Moir R, Wilson A, Stones-Havas S, Cheung M, Sturrock S, et al. Geneious basic: an integrated and extendable desktop software platform for the organization and analysis of sequence data. Bioinformatics. 2012;28(12): 1647-9.

56. Edgar RC. MUSCLE: multiple sequence alignment with high accuracy and high throughput. Nucleic Acids Res. 2004;32(5):1792-7.

57. Gouy M, Guindon S, Gascuel O. SeaView version 4: a multiplatform graphical user interface for sequence alignment and phylogenetic tree building. Mol Biol Evol. 2010;27(2):221-4

58. Talavera G, Castresana J. Improvement of phylogenies after removing divergent and ambiguously aligned blocks from protein sequence alignments. Syst Biol. 2007;56(4):564-77.

59. Guindon S, Lethiec F, Duroux P, Gascuel O. PHYML online--a web server for fast maximum likelihood-based phylogenetic inference. Nucleic Acids Res. 2005;33(Web Server issue):W557-9.

60. Lefort V, Longueville JE, Gascuel O. SMS: smart model selection in PhyML. Mol Biol Evol. 2017;34(9):2422-4.
61. Anisimova M, Gil M, Dufayard JF, Dessimoz C, Gascuel O. Survey of branch support methods demonstrates accuracy, power, and robustness of fast likelihood-based approximation schemes. Syst Biol. 2011;60(5):685-99.

62. Rambaut A, Lam T, Max Carvalho L, Pybus OG. Exploring the temporal structure of heterochronous sequences using TempEst (formerly Path-OGen). Virus Evol. 2016;2(1):vew007.

63. Suchard MA, Lemey P, Baele G, Ayres DL, Drummond AJ, Rambaut A. Bayesian phylogenetic and phylodynamic data integration using BEAST 1. 10. Virus Evol. 2018;4(1):vey016.

64. Rambaut A, Drummond AJ, Xie D, Baele G, Suchard MA. Posterior summarization in Bayesian phylogenetics using tracer 1.7. Syst Biol. 2018; 67(5):901-4

65. Ehlers B, Anoh AE, Ben SN, Broll S, Couacy-Hymann E, Fischer D, et al. Novel polyomaviruses in mammals from multiple orders and reassessment of polyomavirus evolution and taxonomy. Viruses. 2019;11(10):930.

66. Ehlers B, Dural G, Yasmum N, Lembo T, de Thoisy B, Ryser-Degiorgis MP, et al. Novel mammalian herpesviruses and lineages within the Gammaherpesvirinae: cospeciation and interspecies transfer. J Virol. 2008; 82(7):3509-16

67. Cvetko L, Turk N, Markotić A, Milas Z, Margaletić J, Miletić-Medved M, et al. Dual infections with Puumala virus and Leptospira interrogans serovar Lora in a bank vole (Clethrionomys glareolus). Am J Trop Med Hyg. 2006;74(4): 612-4.

68. Schmidt S, Essbauer SS, Mayer-Scholl A, Poppert S, Schmidt-Chanasit J, Klempa B, et al. Multiple infections of rodents with zoonotic pathogens in Austria. Vector Borne Zoonotic Dis. 2014;14(7):467-75.

69. Tadin A, Turk N, Korva M, Margaletić J, Beck R, Vucelja M, et al. Multiple coinfections of rodents with hantaviruses, Leptospira, and Babesia in Croatia Vector-Borne Zoonotic Dis. 2012;12(5):388-92.

70. McAfee MS, Huynh TP, Johnson JL, Jacobs BL, Blattman JN. Interaction between unrelated viruses during in vivo co-infection to limit pathology and immunity. Virology. 2015;484:153-62.

71. Seki M, Yanagihara K, Higashiyama Y, Fukuda Y, Kaneko Y, Ohno H, et al. Immunokinetics in severe pneumonia due to influenza virus and bacteria coinfection in mice. Eur Respir J. 2004;24(1):143-9.

\section{Publisher's Note}

Springer Nature remains neutral with regard to jurisdictional claims in published maps and institutional affiliations.

Ready to submit your research? Choose BMC and benefit from

- fast, convenient online submission

- thorough peer review by experienced researchers in your field

- rapid publication on acceptance

- support for research data, including large and complex data types

- gold Open Access which fosters wider collaboration and increased citations

- maximum visibility for your research: over $100 \mathrm{M}$ website views per year

At $\mathrm{BMC}$, research is always in progress.

Learn more biomedcentral.com/submissions 